\title{
SEARCHING FOR A STOCHASTIC BACKGROUND OF GRAVITATIONAL WAVES WITH THE LASER INTERFEROMETER GRAVITATIONAL-WAVE OBSERVATORY
}

B. Аbbott,${ }^{1}$ R. Авbott, ${ }^{1}$ R. Adhikari, ${ }^{1}$ J. Agresti,${ }^{1}$ P. Ajtth,${ }^{2}$ B. Allen,,${ }^{3}$ R. Amin,${ }^{4}$ S. B. Anderson,${ }^{1}$ W. G. Anderson, ${ }^{3}$ M. Araya,${ }^{1}$ H. Armandula, ${ }^{1}$ M. Ashley, ${ }^{5}$ S Aston, ${ }^{6}$ C. Aulbert,${ }^{7}$ S. Babak, ${ }^{7}$ S. Ballmer,${ }^{8}$ B. C. Barish, ${ }^{1}$ C. Barker,${ }^{9}$ D. Barker, ${ }^{9}$

B. Barr,${ }^{10}$ P. Barriga,${ }^{11}$ M. A. Barton, ${ }^{1}$ K. Bayer,${ }^{8}$ K. Belczynski,${ }^{12}$ J. Betzwieser,${ }^{8}$ P. Beyersdorf, ${ }^{13}$ B. Bhawal, ${ }^{1}$ I. A. Bilenko, ${ }^{14}$ G. Billingsley, ${ }^{1}$ E. Black,${ }^{1}$ K. Blackburn ${ }^{1}$ L. Blackburn,${ }^{8}$ D. Blair, ${ }^{11}$ B. Bland, ${ }^{9}$ L. Bogue,${ }^{15}$ R. Bork, ${ }^{1}$ S. Bose,${ }^{16}$ P. R. Brady, ${ }^{3}$ V. B. Braginsky, ${ }^{14}$ J. E. Brau,${ }^{17}$ A. Brooks,${ }^{18}$ D. A. Brown,,${ }^{1}$ A. Bullington ${ }^{13}$ A. Bunkowski, ${ }^{2}$ A. Buonanno, ${ }^{19}$ R. Burman, ${ }^{11}$ D. Busby, ${ }^{1}$ R. L. Byer,,${ }^{13}$ L. Cadonati, ${ }^{8}$ G. Cagnoli, ${ }^{10}$ J. B. Camp,,${ }^{20}$ J. Cannizzo, ${ }^{20}$ K. Cannon, ${ }^{3}$ C. A. Cantley, ${ }^{10}$ J. Cao,${ }^{8}$ L. Cardenas, ${ }^{1}$ M. M. Casey, ${ }^{10}$ C. Cepeda, ${ }^{1}$ P. Charlton, ${ }^{1}$ S. Chatterji, ${ }^{1}$ S. Chelkowski, ${ }^{2}$ Y. Chen,,${ }^{7}$ D. Chin, ${ }^{21}$ E. Chin, ${ }^{11}$ J. Chow, ${ }^{5}$ N. Christensen, ${ }^{22}$ T. Cokelaer,${ }^{23}$ C. N. Colacino, ${ }^{6}$ R. Coldwell, ${ }^{24}$ D. Cook,${ }^{9}$ T. Corbitt,${ }^{8}$ D. Coward,${ }^{11}$ D. Coyne,${ }^{1}$ J. D. E. Creighton,${ }^{3}$ T. D. Creighton, ${ }^{1}$ D. R. M. Crooks, ${ }^{10}$ A. M. Cruise, ${ }^{6}$ A. Cumming, ${ }^{10}$ C. Cutler, ${ }^{25}$ J. Dalrymple, ${ }^{26}$ E. D'Ambrosio, ${ }^{1}$ K. Danzmann,${ }^{27,2}$ G. Davies, ${ }^{23}$ G. de Vine, ${ }^{5}$ D. DeBra, ${ }^{13}$ J. Degallaix, ${ }^{11}$ V. Dergachev,${ }^{21}$ S. Desai, ${ }^{28}$ R. DeSalvo, ${ }^{1}$ S. Dhurandar, ${ }^{29}$ A. Di Credico, ${ }^{26}$ M. Díaz, ${ }^{30}$ J. Dickson, ${ }^{5}$ G. Diederichs, ${ }^{27}$ A. Dietz, ${ }^{4}$ E. E. Doomes, ${ }^{31}$ R. W. P. Drever, ${ }^{32}$ J.-C. Dumas, ${ }^{11}$ R. J. Dupuis, ${ }^{1}$ P. Ehrens, ${ }^{1}$ E. Elliffe, ${ }^{10}$ T. Etzel, ${ }^{1}$ M. Evans, ${ }^{1}$ T. Evans, ${ }^{15}$ S. Fairhurst, ${ }^{3}$ Y. Fan, ${ }^{11}$ M. M. Fejer, ${ }^{13}$ L. S. Finn, ${ }^{28}$ N. Fotopoulos, ${ }^{8}$ A. Franzen, ${ }^{27}$ K. Y. Franzen,${ }^{24}$ R. E. Frey,${ }^{17}$ T. Fricke, ${ }^{33}$ P. Fritschel,${ }^{8}$ V. V. Frolov, ${ }^{15}$ M. Fyffe, ${ }^{15}$ J. Garofoli, ${ }^{9}$ I. Gholami, ${ }^{7}$ J. A. Giaime, ${ }^{4}$ S. Giampanis, ${ }^{33}$ K. Goda, ${ }^{8}$ E. Goetz,${ }^{21}$ L. Goggin ${ }^{1}$ G. González, ${ }^{4}$ S. Gossler, ${ }^{5}$ A. Grant,${ }^{10}$ S. Gras,${ }^{11}$ C. Gray,${ }^{9}$ M. Gray, ${ }^{5}$ J. Greenhalgh, ${ }^{34}$ A. M. Gretarsson, ${ }^{35}$ D. Grimmett, ${ }^{1}$ R. Grosso, ${ }^{30}$ H. Grote, ${ }^{2}$ S. Grunewald, ${ }^{7}$ M. Guenther, ${ }^{9}$ R. Gustafson, ${ }^{21}$ B. Hage,${ }^{27}$ C. Hanna, ${ }^{4}$ J. Hanson, ${ }^{15}$ C. Hardham,${ }^{13}$ J. Harms,${ }^{2}$ G. Harry, ${ }^{8}$ E. Harstad, ${ }^{17}$ T. Hayler,${ }^{34}$ J. Heefner, ${ }^{1}$ I. S. Heng, ${ }^{10}$ A. Heptonstall,${ }^{10}$ M. Heurs,${ }^{27}$ M. Hewitson, ${ }^{2}$ S. Hild,${ }^{27}$ N. Hindman, ${ }^{9}$ E. Hirose,${ }^{26}$ D. Hoak,${ }^{15}$ P. Hoang, ${ }^{1}$ D. Hosken, ${ }^{18}$ J. Hough,,${ }^{10}$ E. Howell, ${ }^{11}$ D. Hoyland, ${ }^{6}$ W. Hua, ${ }^{13}$ S. Huttner, ${ }^{10}$ D. Ingram, ${ }^{9}$ M. Ito, ${ }^{17}$ Y. Itoh,${ }^{3}$ A. Ivanov, ${ }^{1}$ D. JACKrel,${ }^{13}$ B. Johnson, ${ }^{9}$ W. W. Johnson, ${ }^{4}$ D. I. Jones,,${ }^{10}$ G. Jones, ${ }^{23}$ R. Jones, ${ }^{10}$ L. Ju, ${ }^{11}$ P. Kalmus, ${ }^{36}$ V. Kalogera, ${ }^{12}$ D. KasprzyK, ${ }^{6}$ E. Katsavounidis, ${ }^{8}$ K. Kawabe, ${ }^{9}$ S. Kawamura, ${ }^{37}$ F. Kawazoe ${ }^{37}$ W. Kells, ${ }^{1}$ F. Ya. Khalili, ${ }^{14}$ A. Khan, ${ }^{15}$ C. Kim, ${ }^{12}$ P. King, ${ }^{1}$ S. Klimenko, ${ }^{24}$ K. Kokeyama, ${ }^{37}$ V. Kondrashov, ${ }^{1}$ S. Koranda, ${ }^{3}$ D. Kozak, ${ }^{1}$ B. Krishnan, ${ }^{7}$ P. Kwee,${ }^{27}$ P. K. Lam, ${ }^{5}$ M. Landry,${ }^{9}$ B. Lantz, ${ }^{13}$ A. Lazzarini, ${ }^{1}$ B. Lee,${ }^{11}$ M. Lei,${ }^{1}$ V. Leonhardt, ${ }^{37}$ I. Leonor, ${ }^{17}$ K. Libbrecht, ${ }^{1}$ P. Lindeuist, ${ }^{1}$ N. A. Lockerbie, ${ }^{6}$ M. Lormand, ${ }^{15}$ M. Lubinski, ${ }^{9}$ H. Lück, ${ }^{27,2}$ B. Machenschalk, ${ }^{7}$ M. MacInnis, ${ }^{8}$ M. Mageswaran, ${ }^{1}$ K. Mailand, ${ }^{1}$ M. Malec ${ }^{27}$ V. Mandic, ${ }^{1}$ S. Márka, ${ }^{36}$ J. Markowitz, ${ }^{8}$ E. Maros,${ }^{1}$ I. Martin, ${ }^{10}$ J. N. Marx, ${ }^{1}$ K. Mason, ${ }^{8}$ L. Matone,${ }^{36}$ N. Mavalvala, ${ }^{8}$ R. McCarthy, ${ }^{9}$ D. E. McClelland, ${ }^{5}$ S. C. McGuire, ${ }^{31}$ M. McHugh, ${ }^{38}$ K. McKenzie, ${ }^{5}$ J. W. C. McNabB, ${ }^{28}$ T. Meier, ${ }^{27}$ A. Melissinos, ${ }^{33}$ G. Mendell, ${ }^{9}$ R. A. Mercer,${ }^{24}$ S. Meshrov, ${ }^{1}$ E. Messaritaki, ${ }^{3}$ C. J. Messenger, ${ }^{10}$ D. Meyers, ${ }^{1}$ E. Mikhailov,${ }^{8}$ S. Mitra, ${ }^{29}$ V. P. Mitrofanov, ${ }^{14}$ G. Mitselmakher, ${ }^{24}$

\footnotetext{
${ }^{1}$ Laser Interferometer Gravitational-Wave Observatory, California Institute of Technology, Pasadena, CA.

${ }_{2}$ Albert-Einstein-Institut, Max-Planck-Institut für Gravitationsphysik, Hannover, Germany.

${ }^{3}$ University of Wisconsin-Milwaukee, Milwaukee, WI.

${ }^{4}$ Louisiana State University, Baton Rouge, LA.

5 Australian National University, Canberra, Australia.

${ }_{7}^{6}$ University of Birmingham, Birmingham, UK.

7 Albert-Einstein-Institut, Max-Planck-Institut für Gravitationsphysik, Golm, Germany.

${ }^{8}$ Laser Interferometer Gravitational-Wave Observatory, Massachusetts Institute of Technology, Cambridge, MA.

9 Laser Interferometer Gravitational-Wave Observatory, Hanford Observatory, Richland, WA.

${ }_{10}^{11}$ University of Glasgow, Glasgow, UK.

${ }_{11}$ University of Western Australia, Crawley, Australia.

12 Northwestern University, Evanston, IL.

13 Stanford University, Stanford, CA.

${ }_{14}$ Moscow State University, Moscow, Russia

15 Laser Interferometer Gravitational-Wave Observatory, Livingston Observatory, Livingston, LA.

16 Washington State University, Pullman, WA.

${ }^{17}$ University of Oregon, Eugene, OR.
}

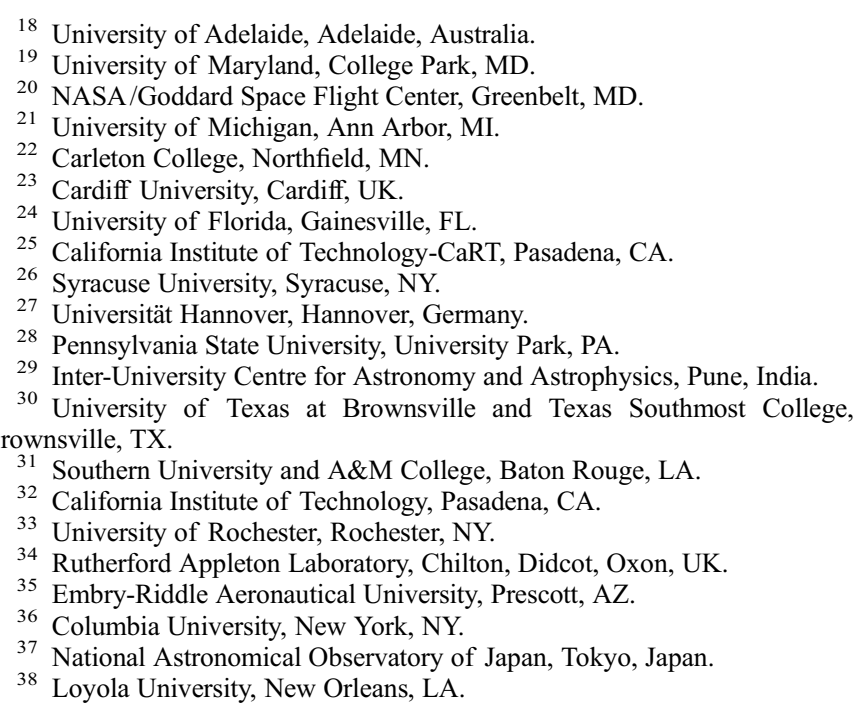


R. Mittleman, ${ }^{8}$ O. Miyakawa ${ }^{1}$ S. Mohanty ${ }^{30}$ G. Moreno, ${ }^{9}$ K. Mossavi,${ }^{2}$ C. Mowlowry, ${ }^{5}$ A. Moylan,${ }^{5}$ D. Mudge, ${ }^{18}$ G. Mueller,${ }^{24}$ H. Müller-Ebhardt ${ }^{2}$ S. Mukherjee, ${ }^{30}$ J. Munch, ${ }^{18}$ P. Murray,${ }^{10}$ E. Myers,${ }^{9}$ J. Myers, ${ }^{9}$ G. Newton, ${ }^{10}$ K. Numata,${ }^{20}$

B. O’Reilly, ${ }^{15}$ R. O'Shaughnessy, ${ }^{12}$ D. J. OTtaway ${ }^{8}$ H. Overmier, ${ }^{15}$ B. J. Owen, ${ }^{28}{ }^{7}$ Y. Pan, ${ }^{25}$ M. A. Papa, ${ }^{7,3}{ }^{70}$

V. Parameshwaraiah, ${ }^{9}$ M. Pedraza, ${ }^{1}$ S. Penn,${ }^{39}$ M. Pitkin,${ }^{10}$ M. V. Plissi, ${ }^{10}$ R. Prix,${ }^{7}$ V. Quetschke ${ }^{24}$ F. Raab, ${ }^{9}$

D. Rabeling,${ }^{5}$ H. Radkins, ${ }^{9}$ R. Rahkola,${ }^{17}$ M. Rakhmanov,${ }^{28}$ K. Rawlins,${ }^{8}$ S. Ray-Majumder, ${ }^{3}$ V. Re, ${ }^{6}$

H. Rehbein, ${ }^{2}$ S. Reid,${ }^{10}$ D. H. Reitze,${ }^{24}$ L. Ribichint, ${ }^{2}$ R. Riesen, ${ }^{15}$ K. Riles, ${ }^{21}$ B. Rivera, ${ }^{9}$ D. I. Robertson, ${ }^{10}$ N. A. Robertson, ${ }^{13,10}$ C. Robinson, ${ }^{23}$ S. Roddy, ${ }^{15}$ A. Rodriguez, ${ }^{4}$ A. M. Rogan, ${ }^{16}$ J. Rollins, ${ }^{36}{ }^{2}$

J. D. Romano ${ }^{23}$ J. Romie, ${ }^{15}$ R. Route, ${ }^{13}$ S. Rowan, ${ }^{10}$ A. Rüdiger, ${ }^{2}$ L. Ruet,${ }^{8}$ P. Russell, ${ }^{1}$ K. Ryan, ${ }^{9}$ S. Sakata,${ }^{37}$ M. Samidi, ${ }^{1}$ L. Sancho de la Jordana,${ }^{40}$ V. Sandberg,,${ }^{9}$ V. Sannibale, ${ }^{1}$ S. Saraf,${ }^{13}$

P. Sarin, ${ }^{8}$ B. S. Sathyaprakash, ${ }^{23}$ S. Sato, ${ }^{37}$ P. R. Saulson, ${ }^{26}$ R. Savage, ${ }^{9}$ S. Schediwy, ${ }^{11}$ R. Schilling, ${ }^{2}$

R. Schnabel, ${ }^{2}$ R. Schofield, ${ }^{17}$ B. F. Schutz, ${ }^{7,23}$ P. Schwinberg, ${ }^{9}$ S. M. Scott,${ }^{5}$ S. E. Seader,,${ }^{16}$

A. C. Searle, ${ }^{5}$ B. Sears, ${ }^{1}$ F. Seifert, ${ }^{2}$ D. Sellers, ${ }^{15}$ A. S. Sengupta,${ }^{23}$ P. Shawhan, ${ }^{1}$ B. Sheard,${ }^{5}$

D. H. Shoemaker, ${ }^{8}$ A. Sibley, ${ }^{15}$ X. Siemens, ${ }^{3}$ D. SigG, ${ }^{9}$ A. M. Sintes, ${ }^{40,7}$ B. Slagmolen, ${ }^{5}$

J. Slutsky, ${ }^{4}$ J. Smith, ${ }^{2}$ M. R. Smith, ${ }^{1}$ P. Sneddon, ${ }^{10}$ K. Somiya, ${ }^{2,7}$ C. Speake, ${ }^{6}$ O. Spjeld, ${ }^{15}$

K. A. Strain, ${ }^{10}$ D. M. Strom,${ }^{17}$ A. Stuver, ${ }^{28}$ T. Summerscales,${ }^{28}$ K. Sun,${ }^{13}$ M. Sung, ${ }^{4}$

P. J. Sutton, ${ }^{1}$ D. B. Tanner, ${ }^{24}$ M. Tarallo, ${ }^{1}$ R. Taylor,${ }^{1}$ R. Taylor,${ }^{10}$ J. Thacker, ${ }^{15}$

K. A. Thorne, ${ }^{28}$ K. S. Thorne, ${ }^{25}$ A. Thüring, ${ }^{27}$ K. V. Tokmakov, ${ }^{14}$ C. Torres,,${ }^{30}$

C. Torrie, ${ }^{1}$ G. Traylor, ${ }^{15}$ M. Trias, ${ }^{40}$ W. Tyler, ${ }^{1}$ D. Ugolini, ${ }^{41}$ C. Ungarelli, ${ }^{6}$

H. Vahlbruch, ${ }^{27}$ M. Vallisneri, ${ }^{25}$ M. Varvella, ${ }^{1}$ S. Vass, ${ }_{9}^{1}$ A. Vecchio, ${ }^{6}$ J. Veitch, ${ }^{10}$ P. Veitch, ${ }^{18}$ S. Vigeland, ${ }^{22}$ A. Villar, ${ }^{1}$ C. Vorvick, ${ }^{9}$ S. P. Vyachanin, ${ }^{14}$

S. J. Waldman, ${ }^{1}$ L. Wallace, ${ }^{1}$ H. Ward,${ }^{10}$ R. Ward,${ }^{1}$ K. Watts, ${ }^{15}$ D. Webber, ${ }^{1}$

A. Weidner, ${ }^{2}$ A. Weinstein, ${ }^{1}$ R. Weiss, ${ }^{8}$ S. Wen, ${ }^{4}$ K. Wette ${ }^{5}$ J. T. Whelan, ${ }^{38,7}$

D. M. Whitbeck ${ }^{28}$ S. E. Whitcomb, ${ }^{1}$ B. F. Whiting, ${ }^{24}$ C. Wilkinson, ${ }^{9}{ }^{8}$

P. A. Willems, ${ }^{1}$ B. Willke, ${ }^{27,2}$ I. Wilmut,${ }^{34}$ W. Winkler, ${ }^{2}$ C. C. WiPf, ${ }^{8}$

S. Wise ${ }^{24}$ A. G. Wiseman, ${ }^{3}$ G. Woan,${ }^{10}$ D. Woods, ${ }^{3}$ R. Wooley,${ }^{15}$

J. Worden, ${ }^{9}$ W. Wu,${ }^{24}$ I. YakUShin, ${ }^{15}$ H. Yamamoto, ${ }^{1}$ Z. Yan,${ }^{11}$

S. Yoshida, ${ }^{42}$ N. Yunes, ${ }^{28}$ M. Zanolin, ${ }^{8}$ L. Zhang, ${ }^{1}$ C. Zhao,${ }^{11}$

N. Zotov ${ }^{43}$ M. ZuCKer, ${ }^{15}$ H. Zur MüHLen, ${ }^{27}$ AND J. Zweizig ${ }^{1}$

(The Ligo Scientific Collaboration) ${ }^{44}$

Received 2006 September 21; accepted 2006 November 30

\begin{abstract}
The Laser Interferometer Gravitational-Wave Observatory (LIGO) has performed the fourth science run, S4, with significantly improved interferometer sensitivities with respect to previous runs. Using data acquired during this science run, we place a limit on the amplitude of a stochastic background of gravitational waves. For a frequency independent spectrum, the new Bayesian $90 \%$ upper limit is $\Omega_{\mathrm{GW}} \times\left[H_{0} /\left(72 \mathrm{~km} \mathrm{~s}^{-1} \mathrm{Mpc}^{-1}\right)\right]^{2}<6.5 \times 10^{-5}$. This is currently the most sensitive result in the frequency range $51-150 \mathrm{~Hz}$, with a factor of 13 improvement over the previous LIGO result. We discuss the complementarity of the new result with other constraints on a stochastic background of gravitational waves, and we investigate implications of the new result for different models of this background.
\end{abstract}

Subject heading: gravitational waves

\section{INTRODUCTION}

A stochastic background of gravitational waves (GWs) is expected to arise as a superposition of a large number of unresolved sources, from different directions in the sky and with different polarizations. It is usually described in terms of the GW spectrum,

$$
\Omega_{\mathrm{GW}}(f)=\frac{f}{\rho_{c}} \frac{d \rho_{\mathrm{GW}}}{d f}
$$

where $d \rho_{\mathrm{GW}}$ is the energy density of gravitational radiation contained in the frequency range $f$ to $f+d f$ (Allen \& Romano

\footnotetext{
39 Hobart and William Smith Colleges, Geneva, NY.

40 Universitat de les Illes Balears, Palma de Mallorca, Spain.

41 Trinity University, San Antonio, TX.

42 Southeastern Louisiana University, Hammond, LA.

43 Louisiana Tech University, Ruston, LA.

44 See http://www.ligo.org.
}

1999), $\rho_{c}$ is the critical energy density of the universe, and $f$ is frequency (for an alternative and equivalent definition of $\Omega_{\mathrm{GW}}(f)$ see, e.g., Baskaran et al. [2006]; Grishchuk et al. [2006]).

Many possible sources of stochastic GW background have been proposed, and several experiments have searched for it (see Maggiore 2000; Allen 1997 for reviews). Some of the proposed theoretical models are cosmological in nature, such as the amplification of quantum vacuum fluctuations during inflation (Grishchuk 1975, 1997; Starobinsky 1979), pre-big bang models (Gasperini \& Veneziano 1993, 2003; Buonanno et al. 1997), phase transitions (Kosowsky et al. 1992; Apreda et al. 2002), and cosmic strings (Caldwell \& Allen 1992; Damour \& Vilenkin 2000, Damour $\&$ Vilenkin 2005). Others are astrophysical in nature, such as rotating neutron stars (Regimbau \& de Freitas Pacheco 2001), supernovae (Coward et al. 2002), or low-mass X-ray binaries (Cooray 2004).

While some of these models predict complex GW spectra, most of them can be well approximated with power laws in the 
Laser Interferometer Gravitational-Wave Observatory (LIGO) frequency band. Hence, we focus on power-law GW spectra:

$$
\Omega_{\mathrm{GW}}(f)=\Omega_{\alpha}\left(\frac{f}{100 \mathrm{~Hz}}\right)^{\alpha}
$$

where $\Omega_{\alpha}$ is the amplitude corresponding to the spectral in$\operatorname{dex} \alpha$. In particular, $\Omega_{0}$ denotes the amplitude of the frequencyindependent $\mathrm{GW}$ spectrum. We consider the range $-3<\alpha<3$.

A number of experiments have been used to constrain the spectrum of GW background at different frequencies. Currently, the most stringent constraints arise from large-angle correlations in the cosmic microwave background (CMB; Allen \& Koranda 1994; Turner 1997), from the arrival times of millisecond pulsar signals (Jenet et al. 2006), from Doppler tracking of the Cassini spacecraft (Armstrong et al. 2003), and from resonant bar GW detectors, such as Explorer and Nautilus (Astone et al. 1999). An indirect bound can be placed on the total energy carried by gravitational waves at the time of the big bang nucleosynthesis (BBN) using the BBN model and observations (Kolb \& Turner 1990; Maggiore 2000; Allen 1997). Similarly, Smith et al. (2006b) used the CMB and matter spectra to constrain the total energy density of gravitational waves at the time of photon decoupling.

Ground-based interferometer networks can directly measure the GW strain spectrum in the frequency band $10 \mathrm{~Hz}$ to a few $\mathrm{kHz}$ by searching for correlated signal beneath uncorrelated detector noise. LIGO has built three power-recycled Michelson interferometers, with a Fabry-Perot cavity in each orthogonal arm. They are located at two sites: Hanford, Washington, and Livingston Parish, Louisiana. There are two collocated interferometers at the Washington site: $\mathrm{H} 1$, with $4 \mathrm{~km}$ long arms, and $\mathrm{H} 2$, with $2 \mathrm{~km}$ arms. The Louisiana site contains L1, a $4 \mathrm{~km}$ interferometer, similar in design to $\mathrm{H} 1$. The detector configuration and performance during LIGO's first science run (S1) was described in Abbott et al. (2004a). The data acquired during that run were used to place an upper limit of $\Omega_{0}<44.4$ on the amplitude of a frequency independent GW spectrum, in the frequency band $40-314 \mathrm{~Hz}$ (Abbott et al. 2004b). For this limit, as well as in the rest of this paper, we assume the present value of the Hubble parameter $H_{0}=$ $72 \mathrm{~km} \mathrm{~s}^{-1} \mathrm{Mpc}^{-1}$ (Bennet et al. 2003), i.e., when writing $\Omega_{0}$, we implicitly mean $\Omega_{0} \times\left[H_{0} /\left(72 \mathrm{~km} \mathrm{~s}^{-1} \mathrm{Mpc}^{-1}\right)\right]^{2}$. The most recent bound on the amplitude of the frequency-independent GW spectrum from LIGO is based on the science run S3; $\Omega_{0}<$ $8.4 \times 10^{-4}$ for a frequency-independent spectrum in the $69-$ $156 \mathrm{~Hz}$ band (Abbott et al. 2005).

In this paper, we report much-improved limits on the stochastic GW background around $100 \mathrm{~Hz}$, using the data acquired during the LIGO science run S4, which took place between 2005 February 22 and March 23. The sensitivity of the interferometers during S4, shown in Figure 1, was significantly better than S3 (by a factor 10 at certain frequencies), which leads to an order-ofmagnitude improvement in the upper limit on the amplitude of the stochastic GW background: $\Omega_{0}<6.5 \times 10^{-5}$ for a frequencyindependent spectrum over the $51-150 \mathrm{~Hz}$ band.

This limit is beginning to probe some models of the stochastic GW background. As examples, we investigate the implications of this limit for cosmic strings models and for pre-big bang models of the stochastic gravitational radiation. In both cases, the new LIGO result excludes parts of the parameter space of these models.

The organization of this paper is as follows. In $\S 2$ we review the analysis procedure and present the results in $\S 3$. In $\S 4$, we discuss some of the implications of our results for models of a stochastic GW background, as well as the complementarity be-

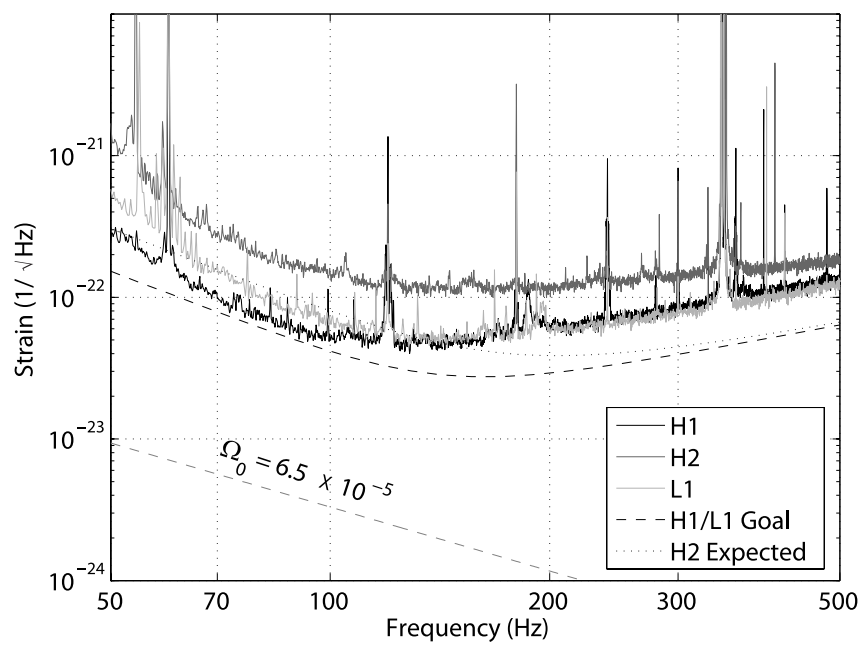

FIG. 1.- Typical strain amplitude spectra of LIGO interferometers during the science run S4 (solid curves top to bottom at $70 \mathrm{~Hz}$ : H2, L1, H1). The black dashed curve is the LIGO sensitivity goal for the $4 \mathrm{~km}$ interferometers H1 and L1. The black dotted curve is the expected design LIGO sensitivity of the $2 \mathrm{~km}$ interferometer H2. The gray dashed curve is the strain amplitude spectrum corresponding to the limit presented in this paper for the frequency-independent GW spectrum $\Omega_{0}<6.5 \times 10^{-5}$.

tween LIGO and other experimental constraints on a stochastic GW background. We conclude with future prospects in $\S 5$.

\section{ANALYSIS}

\subsection{Cross-Correlation Method}

The cross-correlation method for searching for a stochastic GW background with pairs of ground-based interferometers is described in Allen \& Romano (1999). We define the following cross-correlation estimator:

$$
\begin{aligned}
Y & =\int_{0}^{+\infty} d f Y(f) \\
& =\int_{-\infty}^{+\infty} d f \int_{-\infty}^{+\infty} d f^{\prime} \delta_{T}\left(f-f^{\prime}\right) \tilde{s}_{1}(f)^{*} \tilde{s}_{2}\left(f^{\prime}\right) \tilde{Q}\left(f^{\prime}\right),
\end{aligned}
$$

where $\delta_{T}$ is a finite-time approximation to the Dirac delta function, $\tilde{s}_{1}$ and $\tilde{s}_{2}$ are the Fourier transforms of the strain time series of two interferometers, and $\tilde{Q}$ is a filter function. Assuming that the detector noise is Gaussian, stationary, uncorrelated between the two interferometers, and much larger than the GW signal, the variance of the estimator $Y$ is given by

$$
\begin{aligned}
\sigma_{Y}^{2} & =\int_{0}^{+\infty} d f \sigma_{Y}^{2}(f) \\
& \approx \frac{T}{2} \int_{0}^{+\infty} d f P_{1}(f) P_{2}(f)|\tilde{Q}(f)|^{2},
\end{aligned}
$$

where $P_{i}(f)$ are the one-sided power spectral densities (PSDs) of the two interferometers, and $T$ is the measurement time. Optimization of the signal-to-noise ratio leads to the following form of the optimal filter (Allen \& Romano 1999):

$$
\tilde{Q}(f)=\mathcal{N} \frac{\gamma(f) S_{\mathrm{GW}}(f)}{P_{1}(f) P_{2}(f)},
$$




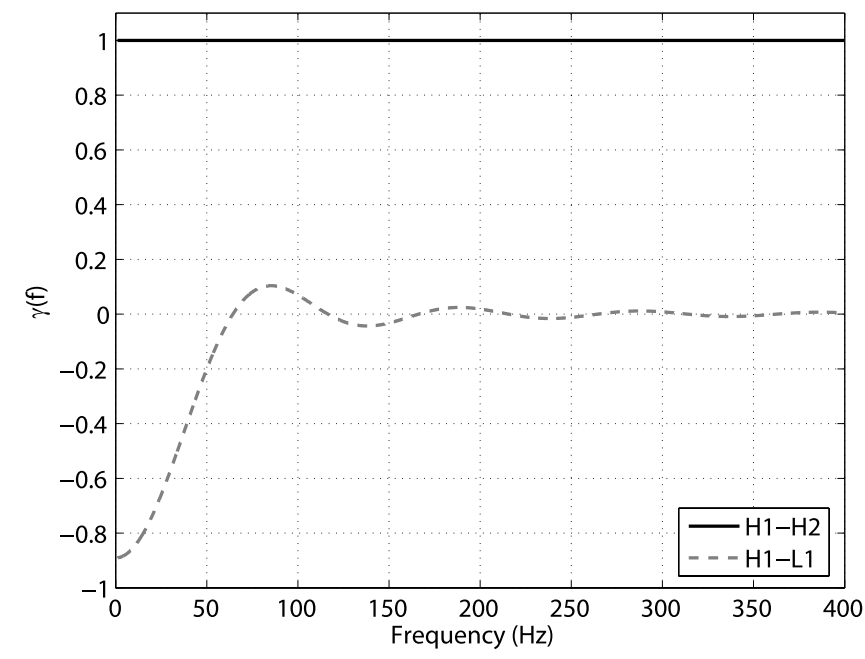

FIG. 2.-Overlap reduction function for the Hanford-Hanford pair (black solid line) and for the Hanford-Livingston pair (gray dashed line).

where

$$
S_{\mathrm{GW}}(f)=\frac{3 H_{0}^{2}}{10 \pi^{2}} \frac{\Omega_{\mathrm{GW}}(f)}{f^{3}}
$$

and $\gamma(f)$ is the overlap reduction function arising from the different locations and orientations of the two interferometers. As shown in Figure 2, the identical antenna patterns of the collocated Hanford interferometers imply $\gamma(f)=1$. For the HanfordLivingston pair the overlap reduction is significant above $50 \mathrm{~Hz}$. In equations (5) and (6), $S_{\mathrm{GW}}(f)$ is the strain power spectrum of the stochastic GW background to be searched. Assuming a powerlaw template GW spectrum with index $\alpha$ (see eq. [2]), the normalization constant $\mathcal{N}$ in equation (5) is chosen such that $\langle Y\rangle=\Omega_{\alpha} T$.

In order to deal with data nonstationarity, and for purposes of computational feasibility, the data for an interferometer pair are divided into many intervals of equal duration, and $Y_{I}$ and $\sigma_{Y_{I}}$ are calculated for each interval $I$. The data in each interval are decimated from 16,384 to $1024 \mathrm{~Hz}$ and high-pass filtered with a $40 \mathrm{~Hz}$ cutoff. As we discuss below, most of the sensitivity of this search lies below $300 \mathrm{~Hz}$, safely below the Nyquist frequency of $512 \mathrm{~Hz}$. The intervals are also Hann windowed to avoid spectral leakage from strong lines present in the data. Since Hann windowing effectively reduces the interval length by $50 \%$, the data intervals are overlapped by $50 \%$ to recover the original signal-tonoise ratio. The effects of windowing are taken into account as discussed in Abbott et al. (2004b).

The PSDs for each interval [needed for the calculation of $Q_{I}(f)$ and of $\left.\sigma_{Y_{I}}\right]$ are calculated using the two neighboring intervals. This approach avoids a bias that would otherwise exist due to a nonzero covariance between the cross-power $Y(f)$ and the power spectra estimated from the same data (Bendat \& Piersol 2000). It also allows for a stationarity cut, which we describe in more detail below.

We consider two interval durations and frequency resolutions:

1. $60 \mathrm{~s}$ duration with $1 / 4 \mathrm{~Hz}$ resolution.-The PSDs are calculated by averaging $5850 \%$ overlapping periodograms (based on the two neighboring $60 \mathrm{~s}$ intervals) in Welch's modified periodogram method.

2. 192 s duration with $1 / 32 \mathrm{~Hz}$ resolution.-The PSDs are calculated by averaging $2250 \%$ overlapping periodograms (based on the two neighboring $192 \mathrm{~s}$ intervals) in Welch's modified periodogram method.

As we discuss below, the $60 \mathrm{~s}$ intervals allow better sensitivity to noise transients and are better suited for data-stationarity cuts. We used this interval duration in the blind analysis. However, after unblinding we discovered a comb of correlated sharp $1 \mathrm{~Hz}$ harmonic lines between interferometers. To remove these sharp lines from our analysis without significantly affecting the sensitivity, we performed the second analysis with $192 \mathrm{~s}$ intervals. The $192 \mathrm{~s}$ intervals allow higher frequency resolution of the power and cross-power spectra and are better suited for removing sharp lines from the analysis.

The data for a given interval $I$ are Fourier transformed (with frequency resolution $1 / 60$ or $1 / 192 \mathrm{~Hz}$ ) and rebinned to the frequency resolution of the PSDs and of the optimal filter (1/4 or $1 / 32 \mathrm{~Hz}$, respectively) to complete the calculation of $Y_{I}$ (eq. [3]). Both the PSDs and the Fourier transforms of the data are calibrated using interferometer response functions, determined for every minute of data using a measurement of the interferometer response to a sinusoidal calibration force. To maximize the signal-to-noise ratio, the intervals are combined by performing a weighted average (with weights $1 / \sigma_{Y_{I}}^{2}$ ), properly accounting for the $50 \%$ overlapping as discussed in Lazzarini \& Romano (2004).

\subsection{Identification of Correlated Instrumental Lines}

The results of this paper are based on the Hanford-Livingston interferometer pairs, for which the broadband instrumental correlations are minimized. Nevertheless, it is still necessary to investigate whether there are any remaining periodic instrumental correlations. We do this by calculating the coherence over the whole S4 run. The coherence is defined as

$$
\Gamma(f)=\frac{\left|P_{12}(f)\right|^{2}}{P_{1}(f) P_{2}(f)} .
$$

The numerator is the square of the cross-spectral density (CSD) between the two interferometers, and the denominator contains the two power spectral densities (PSDs). We average the CSD and the PSDs over the whole run at two different resolutions: 1 and $100 \mathrm{mHz}$. Figure 3 shows the results of this calculation for the H1-L1 pair and for the H2-L1 pair.

At $1 \mathrm{mHz}$ resolution, a forest of sharp $1 \mathrm{~Hz}$ harmonic lines can be observed. These lines were likely caused by the sharp ramp of a one-pulse-per-second signal, injected into the data acquisition system to synchronize it with the Global Positioning System (GPS) time reference. Since the coupling of this signal to the gravitational-wave channel of $\mathrm{H} 1$ was much stronger than that of $\mathrm{H} 2$, the forest of $1 \mathrm{~Hz}$ harmonics is much clearer in the H1-L1 coherence. After the S4 run ended, the sharp ramp signal was replaced by smooth sinusoidal signals, with the goal of significantly reducing the $1 \mathrm{~Hz}$ harmonic lines in future LIGO data runs. In addition to the $1 \mathrm{~Hz}$ lines, the $1 \mathrm{mHz}$ coherence plots in Figure 3 also include some of the simulated pulsar lines, which were injected into the differential-arm servo of the interferometers by physically moving the mirrors. Both the $1 \mathrm{~Hz}$ harmonics and the simulated pulsar lines can be removed in the final analysis, and we discuss this further in $\S 3$. Figure 4 shows that the histogram of the coherence at $1 \mathrm{mHz}$ resolution follows the expected exponential distribution, if one ignores the $1 \mathrm{~Hz}$ harmonics and the simulated pulsar lines. 

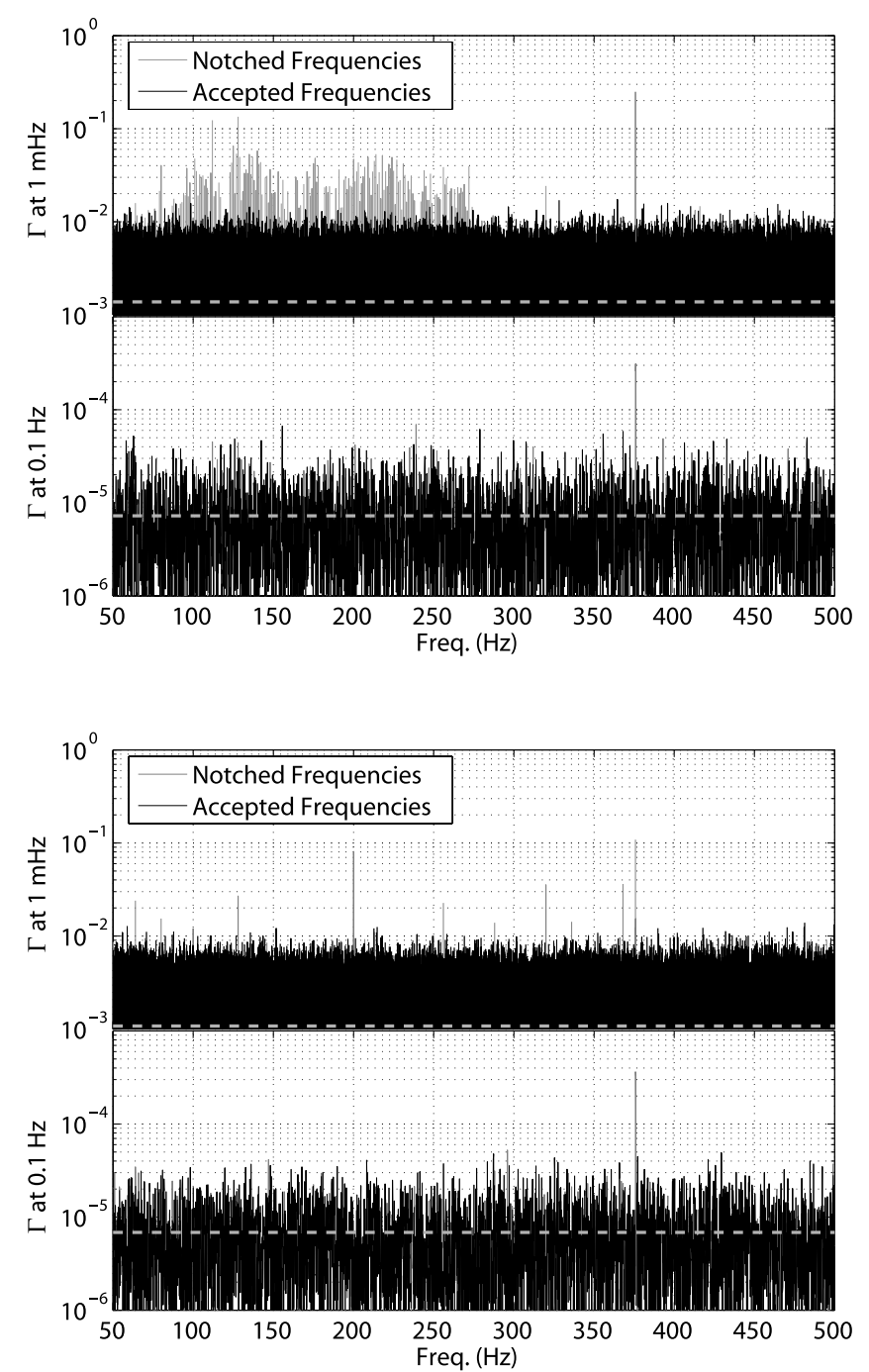

FIG. 3.-Coherence calculated for the H1-L1 pair (top) and for the H2-L1 pair (bottom) over all of S4 data for 1 and $100 \mathrm{mHz}$ resolution. The horizontal dashed lines indicate $1 / N_{\text {avg }}$, the expected level of coherence after averaging over $N_{\text {avg }}$ time periods with uncorrelated spectra. The line at $376 \mathrm{~Hz}$ is one of the simulated pulsar lines.

\subsection{Data Quality Cuts}

In our analysis, we include time periods during which both interferometers are in low-noise, science mode. We exclude:

1. Time periods when digitizer signals saturate.

2. $30 \mathrm{~s}$ intervals prior to each "lock loss"; interferometers become increasingly unstable during these intervals, usually due to some external disturbance, until they eventually fail to maintain the fixed distances between mirrors.

We then proceed to calculate $Y_{I}$ and $\sigma_{Y_{I}}$ for each interval $I$ and define three data quality cuts. First, we reject intervals known to contain large glitches in one interferometer. These intervals were identified by searching for discontinuities in the PSD trends over the whole S4 run. Second, we reject intervals for which $\sigma_{Y_{I}}$ is anomalously large. This cut is designed to reject intervals that happen during particularly noisy periods, by rejecting about $2 \%$ of the intervals in the high-end tail of the $\sigma_{Y_{I}}$ distribution. In particular, for the $192 \mathrm{~s}$ analysis, we require $\sigma_{Y_{I}}<1 \mathrm{~s}$ for the H1-L1 pair, and $\sigma_{Y_{I}}<2 \mathrm{~s}$ for the H2-L1 pair (recall that $Y$ is normalized such that $\langle Y\rangle=\Omega_{\alpha} T$, with $T=192 \mathrm{~s}$ in this case). The glitch cut and the large $\sigma$ cut largely overlap and are designed to remove
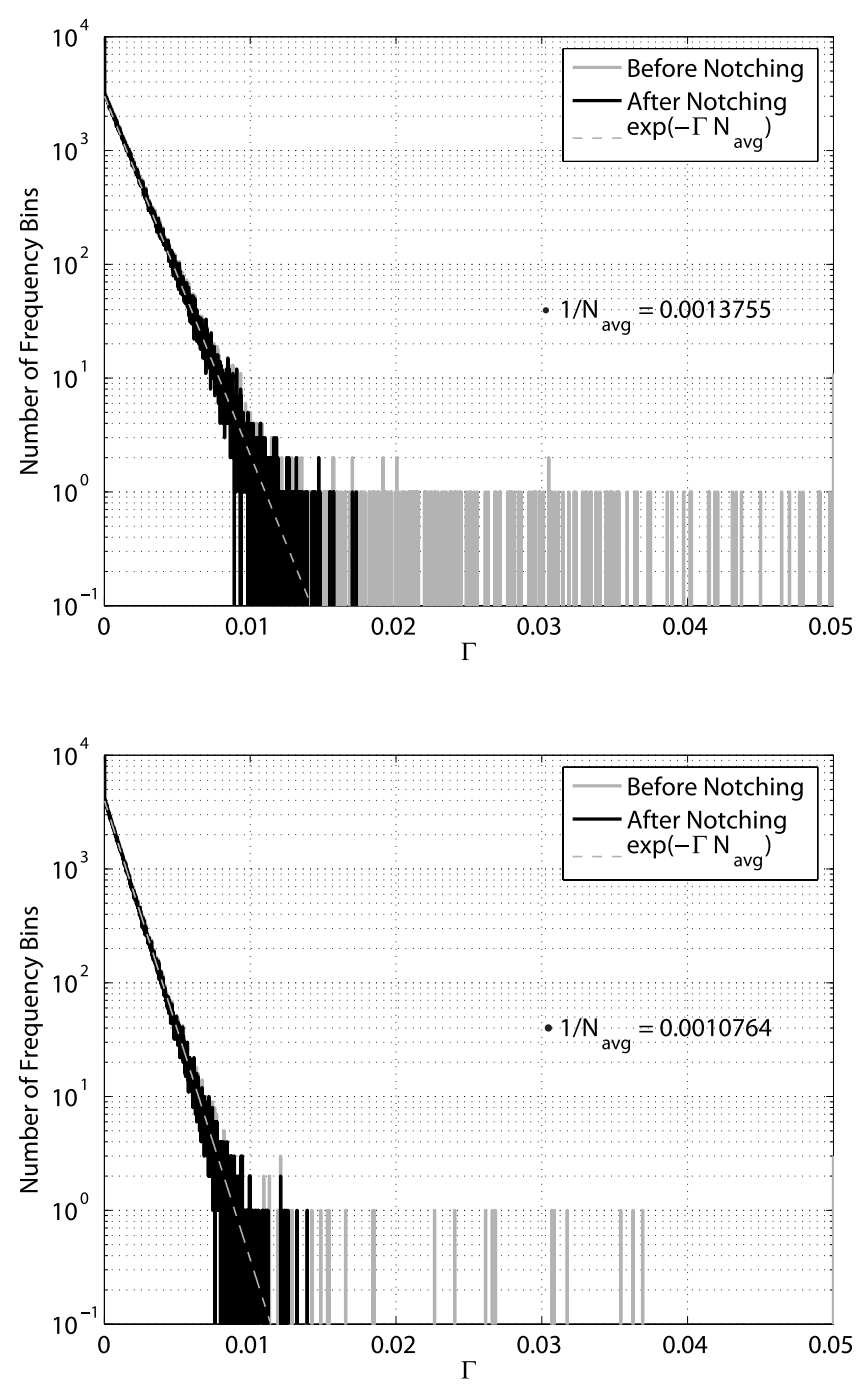

FIG. 4.- Histogram of the coherence for H1-L1 (top) and H2-L1 (bottom) at $1 \mathrm{mHz}$ resolution. The histogram follows the expected exponential distribution, with exponent coefficient $N_{\text {avg }}$ (the number of time periods over which the average is made), when the $1 \mathrm{~Hz}$ harmonics are removed ("after notching"). The gray histograms ("before notching") show the outliers from the exponential distribution due to the $1 \mathrm{~Hz}$ harmonics.

particularly noisy time periods from the analysis. Note also that due to the weighting with $1 / \sigma_{Y_{I}}^{2}$, the contribution of these intervals to the final result would be suppressed, but we reject them from the analysis nevertheless. Third, we reject the intervals for which $\Delta \sigma=\left|\sigma_{Y_{I}}-\sigma_{Y_{I}}^{\prime}\right| / \sigma_{Y_{I}}>\zeta$. Here, $\sigma_{Y_{I}}$ is calculated using the two intervals neighboring interval $I$, and $\sigma_{Y_{I}}^{\prime}$ is calculated using the interval $I$ itself. The optimization of threshold $\zeta$ is discussed below. The goal of this cut is to capture noise transients in the data and reject them from the analysis. Figure 5 shows the impact of these cuts for the H1-L1 pair, analyzed with $192 \mathrm{~s}$ segments, $1 / 32 \mathrm{~Hz}$ resolution, and with $\zeta=0.3$. This figure also shows daily variation in the sensitivity to stochastic GW background, arising from the variation in the strain sensitivity of the interferometers, which is typically worse during the weekdays than during the weekends or nights.

Figure 6 shows the distribution of the residuals for the same analysis. For a given interval $I$, the residual is defined as

$$
\frac{Y_{I}-\langle Y\rangle}{\sigma_{Y_{I}}},
$$




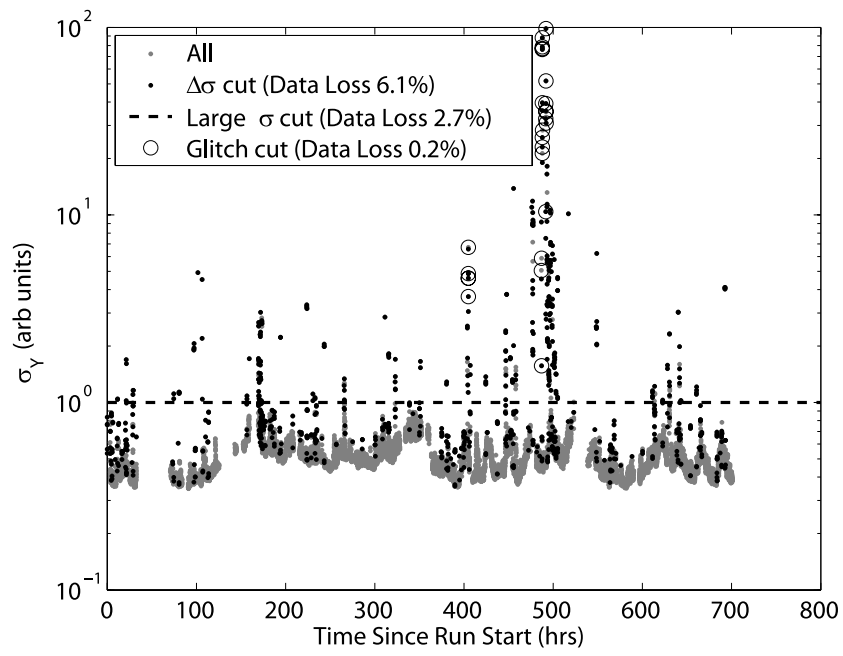

FIG. 5.- Trend of $\sigma_{Y_{I}}$ over the whole S4 run for the $192 \mathrm{~s}$ intervals of H1-L1 pair. The dashed line denotes the large $\sigma$ cut; segments lying above this line are removed from analysis. Note the daily variation in the sensitivity of this pair.

where $\langle Y\rangle$ is calculated using only the data that pass all the data quality cuts. Also note that the data quality cuts remove outliers from the residual distribution, hence making the data more stationary. After the cuts, the Kolmogorov-Smirnov test indicates that the residual distribution is consistent with a Gaussian, for both H1-L1 and H2-L1 analyses with $192 \mathrm{~s}$ intervals, 1/32 Hz resolution, and $\zeta=0.3$.

\section{RESULTS}

\subsection{New Upper Limit}

We performed a "blind" analysis for the H1-L1 and the $\mathrm{H} 2-\mathrm{L} 1$ pairs with $60 \mathrm{~s}$ intervals, $1 / 4 \mathrm{~Hz}$ resolution, and $\zeta=0.2$. To avoid biasing the results, all data quality cuts were defined based on studies done with a $0.1 \mathrm{~s}$ time shift between the two interferometers in a pair. Such a time shift removes any GW correlations, without significantly affecting the instrumental noise performance. After the data quality cuts were finalized, we made one last pass through the data, with zero time shift, and obtained the final results of the blind analysis.

The results from the blind analysis for the frequency-independent template spectrum $(\alpha=0)$ are listed in the first row of Table 1 for H1-L1 and in the first row of Table 2 for H2-L1. These results show no evidence of a stochastic GW background. After completing the blind analysis, we discovered that the instrumental $1 \mathrm{~Hz}$ harmonic lines, discussed in $\S 2.2$, are correlated between the two sites. We felt compelled on scientific grounds not to ignore these correlations, even though they had been discovered after our initial, blind, analysis was complete.

In order to remove from our results any possible influence of the correlated lines, we repeated our analysis with refined frequency resolution of $1 / 32 \mathrm{~Hz}$. We increased the interval length from 60 to $192 \mathrm{~s}$, which implies that the PSDs are estimated by averaging $2250 \%$ overlapping periodograms. These changes allowed us to exclude the $1 \mathrm{~Hz}$ harmonics from the analysis while losing only $\sim 3 \%$ of the bandwidth. Besides the low cost in bandwidth, the advantages of this approach are its simplicity and the fact that it does not introduce new systematics effects. The drawback, however, was that the $192 \mathrm{~s}$ analysis was less able to identify and exclude the noise transients than the $60 \mathrm{~s}$ analysis, as shown in Figure 7. As a result, the $\Delta \sigma$ cut was retuned for the

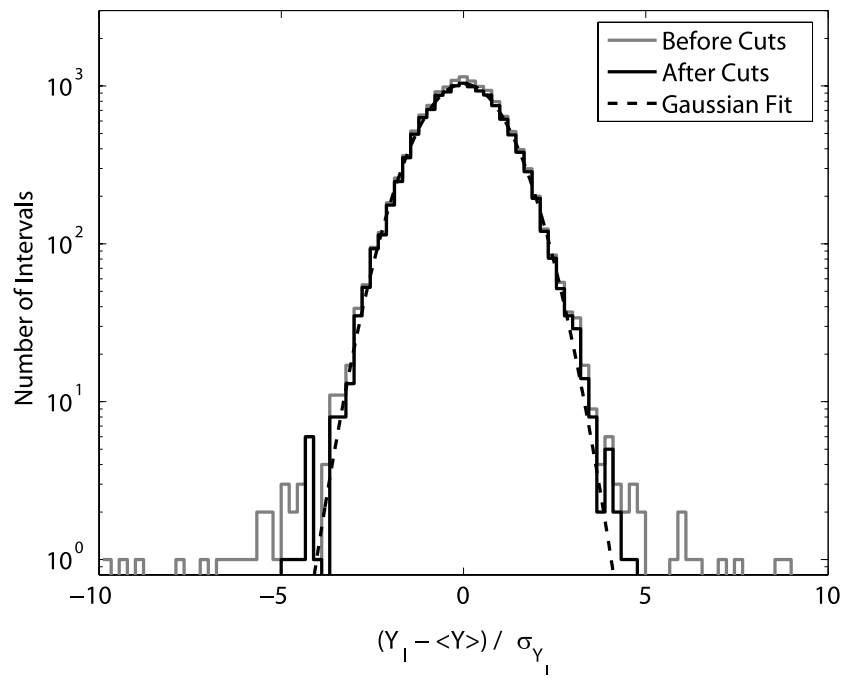

FIG. 6.-Distribution of residuals for the H1-L1 pair with $192 \mathrm{~s}$ segments. All data are shown in gray, data that pass data quality cuts are shown in black, and the Gaussian fit to the black histogram is shown as a dashed curve.

$192 \mathrm{~s}$ analysis after the $60 \mathrm{~s}$ analysis with zero time shift was completed.

We examined several methods of defining the data quality cuts for the $192 \mathrm{~s}$ analysis. First, we calculated the $\Delta \sigma$ cut in the $60 \mathrm{~s}$ analysis with $\zeta=0.2$ (along with large $\sigma$ and glitch cuts), and then declared $192 \mathrm{~s}$ intervals "bad" if they overlapped with the "bad" $60 \mathrm{~s}$ intervals. This approach has the advantage that the $60 \mathrm{~s}$ analysis is more sensitive to transients, and the disadvantage that a significant fraction of the data is rejected. The results of this approach are summarized in the second row of Tables 1 and 2. Second, we defined all cuts directly on $192 \mathrm{~s}$ intervals. We varied the value of $\zeta(0.2,0.3$, and 0.4$)$ and selected $\zeta=0.3$ as the optimal parameter choice. This choice represents the best compromise between data loss and data quality; it rejects the least amount of data, while still excluding all residual outliers and preserving the Gaussianity of the data. The last three rows of Tables 1 and 2 summarize the results for the three values of $\zeta$.

The $192 \mathrm{~s}$ analysis was not performed blindly. However, it agrees with our blind $60 \mathrm{~s}$ analysis very well, because the contribution of the correlated $1 \mathrm{~Hz}$ harmonic lines is only about $1.5 \sigma$. It is also more conservative than the blind analysis, since the value of the theoretical error is larger due to a smaller amount of data available in the form of acceptable $192 \mathrm{~s}$ intervals (as compared to the $60 \mathrm{~s}$ intervals). It also properly handles the known instrumental correlations at $1 \mathrm{~Hz}$ harmonics. Hence, in the remaining part of the paper we focus on the $192 \mathrm{~s}$ analysis with $\zeta=0.3$. For the H1-L1 pair, 12,637 50\% overlapping $192 \mathrm{~s}$ intervals pass all data quality cuts of this analysis, amounting to $353.9 \mathrm{hr}$ of exposure. For the H2-L1 pair, 11,849 intervals pass all data quality cuts of this analysis, amounting to $332.7 \mathrm{hr}$ of exposure.

Figures 8 and 9 show the results of the $192 \mathrm{~s}$ analysis with $\zeta=0.3$ for H1-L1 and H2-L1, respectively. The top plots in these two figures show the cumulative estimates for the frequencyindependent $\mathrm{GW}$ template $(\alpha=0)$ as a function of time. They indicate that there is no particular time during the run that dominates the result. Moreover, the $\pm 1.65 \sigma$ bounds converge as $\sim 1 / \sqrt{T}$, as expected. The middle plots of the two figures show the cross-correlation spectra (i.e., the integrand of eq. [3]). They indicate that there is no particular frequency that dominates the result. 
TABLE 1

H1-L1 RESULTS

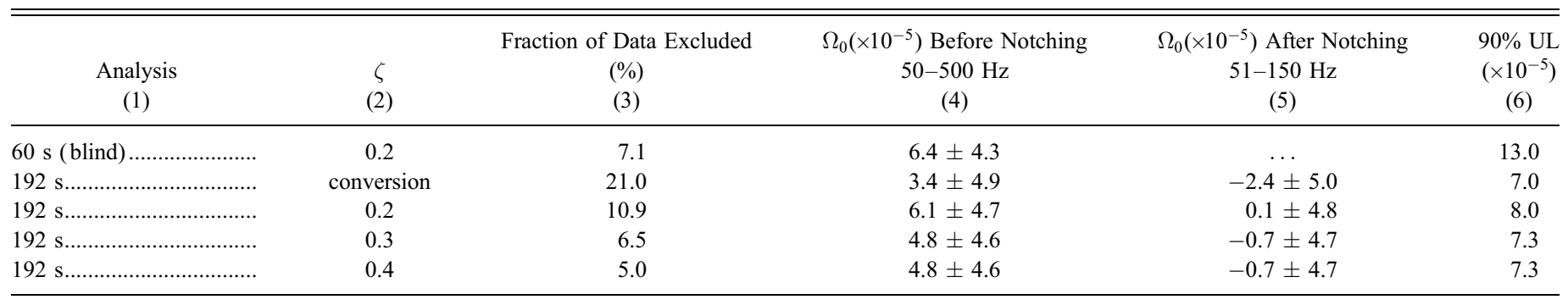

Notes.-H1-L1 results, for $H_{0}=72 \mathrm{~km} \mathrm{~s}^{-1} \mathrm{Mpc}^{-1}$ and for a frequency-independent template spectrum $(\alpha=0)$. Cols. (1) and (2) define the analysis (interval duration and $\Delta \sigma$ cut). For the $192 \mathrm{~s}$ analysis with $\zeta$ denoted as "conversion" (second row), the $\Delta \sigma$ cut was defined using the $60 \mathrm{~s}$ analysis listed in the first row: the $192 \mathrm{~s}$ segments are declared "bad" if they overlap with a "bad" $60 \mathrm{~s} \mathrm{segment.} \mathrm{Col.} \mathrm{(3):} \mathrm{Fraction} \mathrm{of} \mathrm{the} \mathrm{data} \mathrm{lost} \mathrm{to} \mathrm{data} \mathrm{quality} \mathrm{cuts.} \mathrm{Col.} \mathrm{(4):} \mathrm{Estimates} \mathrm{of} \Omega_{0}$ for the $50-500 \mathrm{~Hz}$ range before notching the $1 \mathrm{~Hz}$ harmonics and the simulated pulsar lines. Col. (5): Estimates of $\Omega_{0}$ for the $51-150 \mathrm{~Hz}$ range after notching the $1 \mathrm{~Hz}$ harmonics and the simulated pulsar lines; this frequency range was determined to be optimal for the combined H1-L1+H2-L1 analysis (see text). Col. (6): The 90\% upper limit based on the result in the col. (5). For all analyses presented here, the distribution of the residual outliers was consistent with a Gaussian distribution (e.g., Fig. 6; the KolmogorovSmirnov test statistic for comparing the two distributions was greater than $85 \%$ in all cases).

We perform a weighted average of the H1-L1 and H2-L1 results at each frequency bin (with inverse variances as weights). The resulting cross-correlation spectrum and the theoretical error are shown in Figure 10. The frequency range $51-150 \mathrm{~Hz}$ contributes $99 \%$ of the full sensitivity, as determined by the inverse combined variance. Integrating over this frequency range, we obtain the final estimate for the frequency-independent spectrum: $\Omega_{0}=(-0.8 \pm 4.3) \times 10^{-5}$.

The dominant systematic uncertainty of this result comes from the amplitude calibration uncertainty of the interferometers. This uncertainty is estimated to be $5 \%(\mathrm{~L} 1)$ and $8 \%(\mathrm{H} 1$ and $\mathrm{H} 2)$, and it is frequency-independent. The uncertainty in the phase of the interferometer strain response is negligible compared to the magnitude and statistical uncertainties. Similarly, the effect of timing errors, measured to be $\leqslant 4 \mu \mathrm{s}$, is negligible. Using hardware injections, we estimate that the effect of the timing errors on our point estimate is $\lesssim 0.2 \%$.

We then construct the Bayesian posterior distribution for $\Omega_{0}$ using the above estimate, following Loredo (1990). We assume a Gaussian distribution for the amplitude calibration uncertainty [with mean 1 and standard deviation $\left(0.05^{2}+0.08^{2}\right)^{1 / 2}=0.093$ ], and we marginalize over it. We assume the prior distribution for $\Omega_{0}$ to be the posterior distribution obtained in our previous analysis of the S3 data (Abbott et al. 2005). The 90\% upper limit is the value of $\Omega_{0}$ for which $90 \%$ of the posterior distribution lies between 0 and the upper limit. This procedure yields the Bayesian
$90 \%$ upper limit (UL) on $\Omega_{0}$ of $6.5 \times 10^{-5}$. This is an improvement by a factor 13 over the previous result in the same frequency band, established based on the science run S3 (Abbott et al. 2005). To investigate robustness of our result under different priors, we repeated the calculation using flat priors for the amplitude calibration uncertainties and using a flat prior for $\Omega_{0}$ between 0 and $8.4 \times 10^{-4}$ (previous $90 \%$ UL by LIGO; Abbott et al. 2005). We found that these different choices of priors have less than $3 \%$ effect on the $90 \%$ upper limit.

Once the estimate is made for the frequency-independent spectrum, one can perform appropriate frequency-dependent scalings of $Y(f)$ and $\sigma_{Y}(f)$, recalculate the posterior distributions, and remarginalize to obtain upper limits for other templates, such as the power-law templates with different spectral indices $\alpha$ (see eq. [2]). Figure 11 shows the $90 \%$ UL as a function of the spectral index $\alpha$ obtained for this analysis. Similar results for the S3 run of LIGO, as well as the expected sensitivities of H1-L1 and $\mathrm{H} 1-\mathrm{H} 2$ pairs assuming $1 \mathrm{yr}$ of exposure and design interferometer sensitivities, are also shown. The frequency range of interest is defined to include $99 \%$ of the full sensitivity, as determined by the inverse variance. For the $\mathrm{S} 4$ result, the frequency range varies between $50-107 \mathrm{~Hz}$ for $\alpha=-3$ and $73-288 \mathrm{~Hz}$ for $\alpha=+3$, as shown in the bottom plot of Figure 11. Note that the expected sensitivity of the collocated Hanford interferometer pair (H1-H2) is significantly better than that of the H1-L1 pair due to the better antenna pattern overlap (see Fig. 2). However, this

TABLE 2

H2-L1 Results

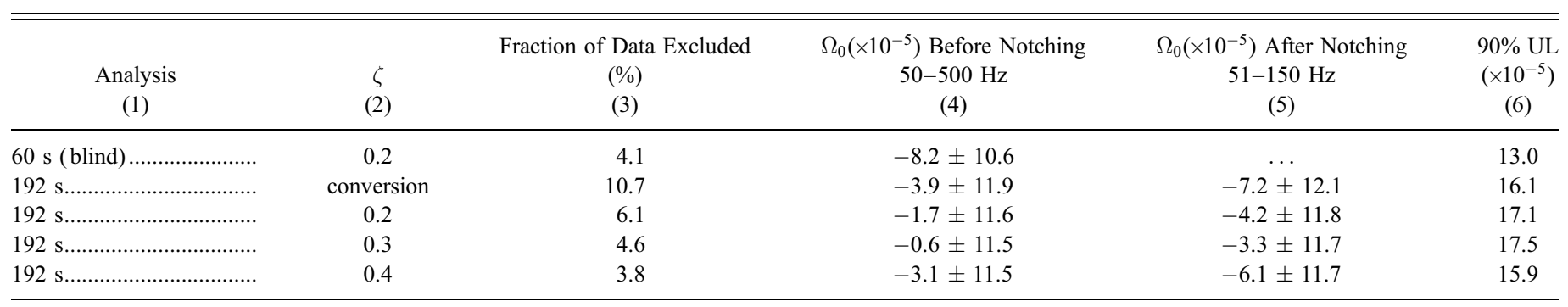

Notes.-H2-L1 results, for $H_{0}=72 \mathrm{~km} \mathrm{~s}^{-1} \mathrm{Mpc}^{-1}$ and for a frequency-independent template spectrum $(\alpha=0)$. Cols. (1) and (2) define the analysis (interval duration and $\Delta \sigma$ cut). For the $192 \mathrm{~s}$ analysis with $\zeta$ denoted as "conversion" (second row), the $\Delta \sigma$ cut was defined using the $60 \mathrm{~s}$ analysis listed in the first row: the $192 \mathrm{~s}$ segments are declared "bad" if they overlap with a "bad" $60 \mathrm{~s} \mathrm{segment.} \mathrm{Col.} \mathrm{(3):} \mathrm{Fraction} \mathrm{of} \mathrm{the} \mathrm{data} \mathrm{lost} \mathrm{to} \mathrm{data} \mathrm{quality} \mathrm{cuts.} \mathrm{Col.} \mathrm{(4):} \mathrm{Estimates} \mathrm{of} \Omega_{0}$ for the $50-500 \mathrm{~Hz}$ range before notching the $1 \mathrm{~Hz}$ harmonics and the simulated pulsar lines. Col. (5): Estimates of $\Omega_{0}$ for the $51-150 \mathrm{~Hz}$ range after notching the $1 \mathrm{~Hz}$ harmonics and the simulated pulsar lines; this frequency range was determined to be optimal for the combined H1-L1+H2-L1 analysis (see text). Col. (6): The 90\% upper limit based on the result in col. (5). For all analyses presented here, the distribution of the residual outliers was consistent with a Gaussian distribution (the Kolmogorov-Smirnov test statistic for comparing the two distributions was greater than $52 \%$ in all cases). 


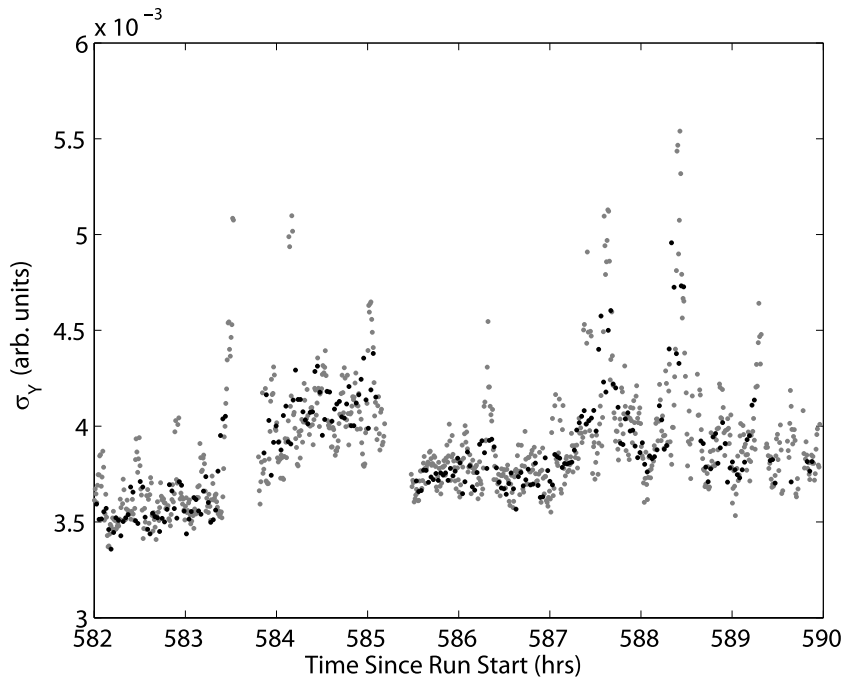

FIG. 7.- Trend of $\sigma_{Y}$ for the $60 \mathrm{~s}$ analysis (gray) and $192 \mathrm{~s}$ analysis (black) over a short period of time. The two bands were scaled to overlap. Note that the gray band is wider, indicating that the $60 \mathrm{~s}$ analysis is more sensitive to noise variations.

pair is also more susceptible to instrumentally correlated noise. New analysis methods are being pursued to estimate and suppress these instrumental correlations (Fotopoulos 2006).

\subsection{Signal Injections}

We exercise the analysis procedure described above using simulated stochastic signals injected into the data both in soft-
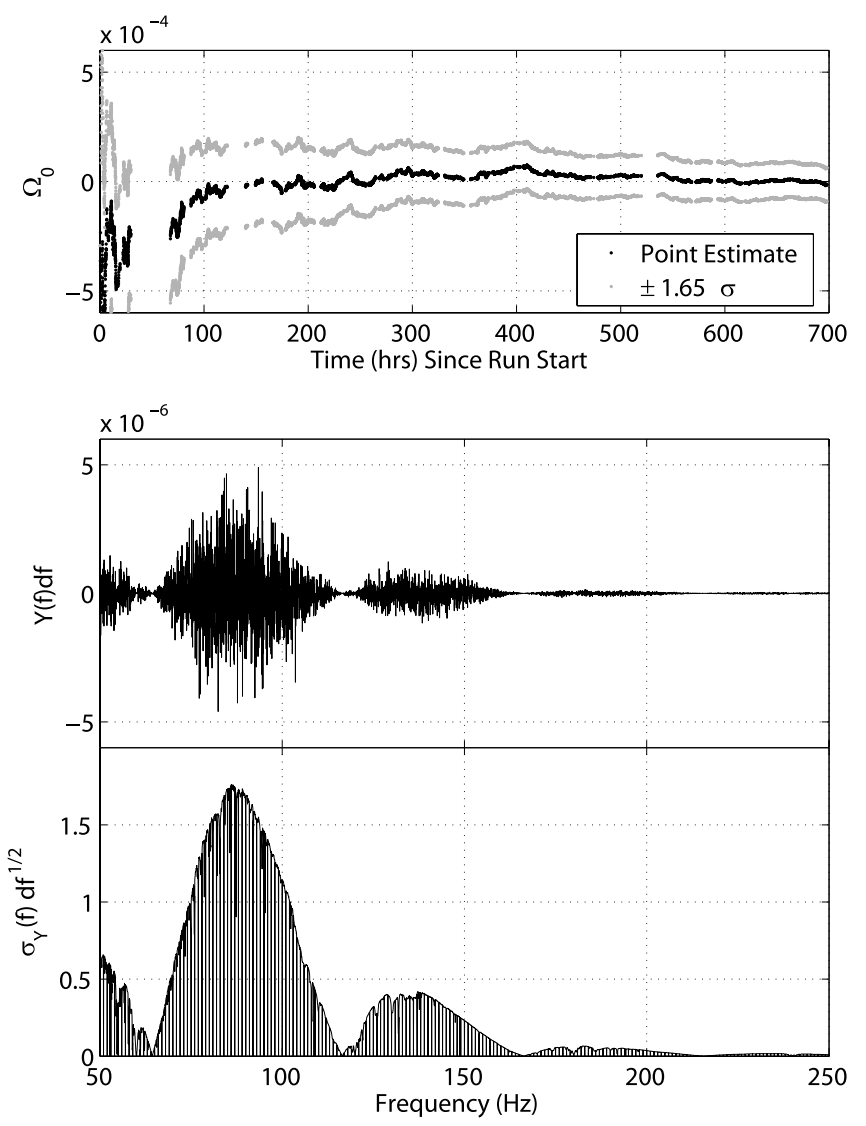

FIG. 8.-H1-L1, $192 \mathrm{~s}$ analysis with $\zeta=0.3$. Top: Cumulative estimate of $\Omega_{0}$ is shown as a function of time. Middle: Cross-correlation spectrum $Y(f)$. Bottom: Theoretical uncertainty $\sigma_{Y}(f)$ as a function of frequency.
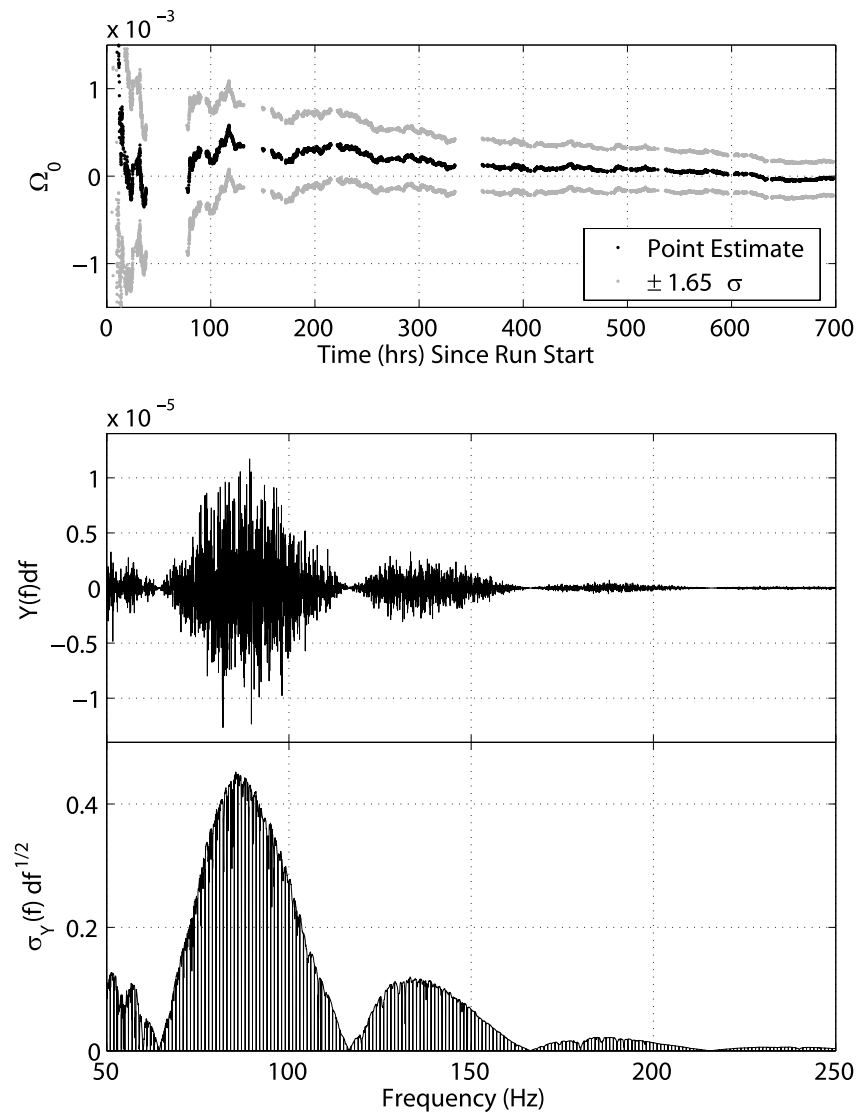

FIG. 9.-H2-L1, $192 \mathrm{~s}$ analysis with $\zeta=0.3$. Top: Cumulative estimate of $\Omega_{0}$ is shown as a function of time. Middle: Cross-correlation spectrum $Y(f)$. Bottom: Theoretical uncertainty $\sigma_{Y}(f)$ as a function of frequency.

ware and in hardware (see Bose et al. 2003). In particular, we verify that the recovery of the injected signals is not affected by the data quality cuts we impose. The hardware injections are performed by physically moving the mirrors to simulate a stochastic GW signal. Three hardware injections were performed during the $\mathrm{S} 4$ run, all using a frequency-independent GW spectrum $(\alpha=0)$. Table 3 summarizes the recovery of all of the hardware injections. Figure 12 shows the cross-correlation spectrum
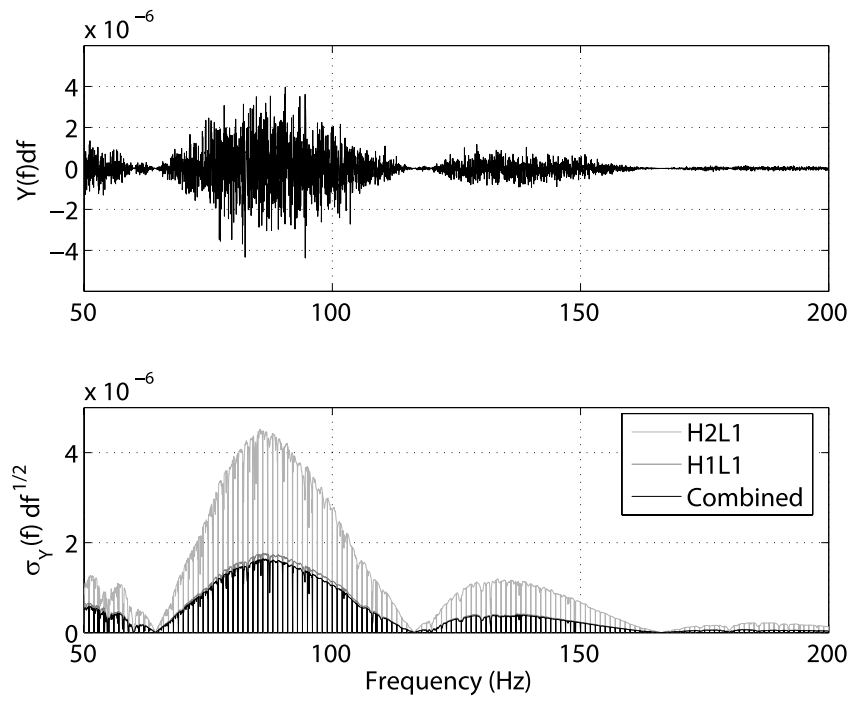

FIG. 10.-Combined H1-L1+H2-L1 result, $192 \mathrm{~s}$ analysis with $\zeta=0.3$. Top: Combined cross-correlation spectrum. Bottom: Theoretical uncertainty $\sigma_{Y}(f)$. 

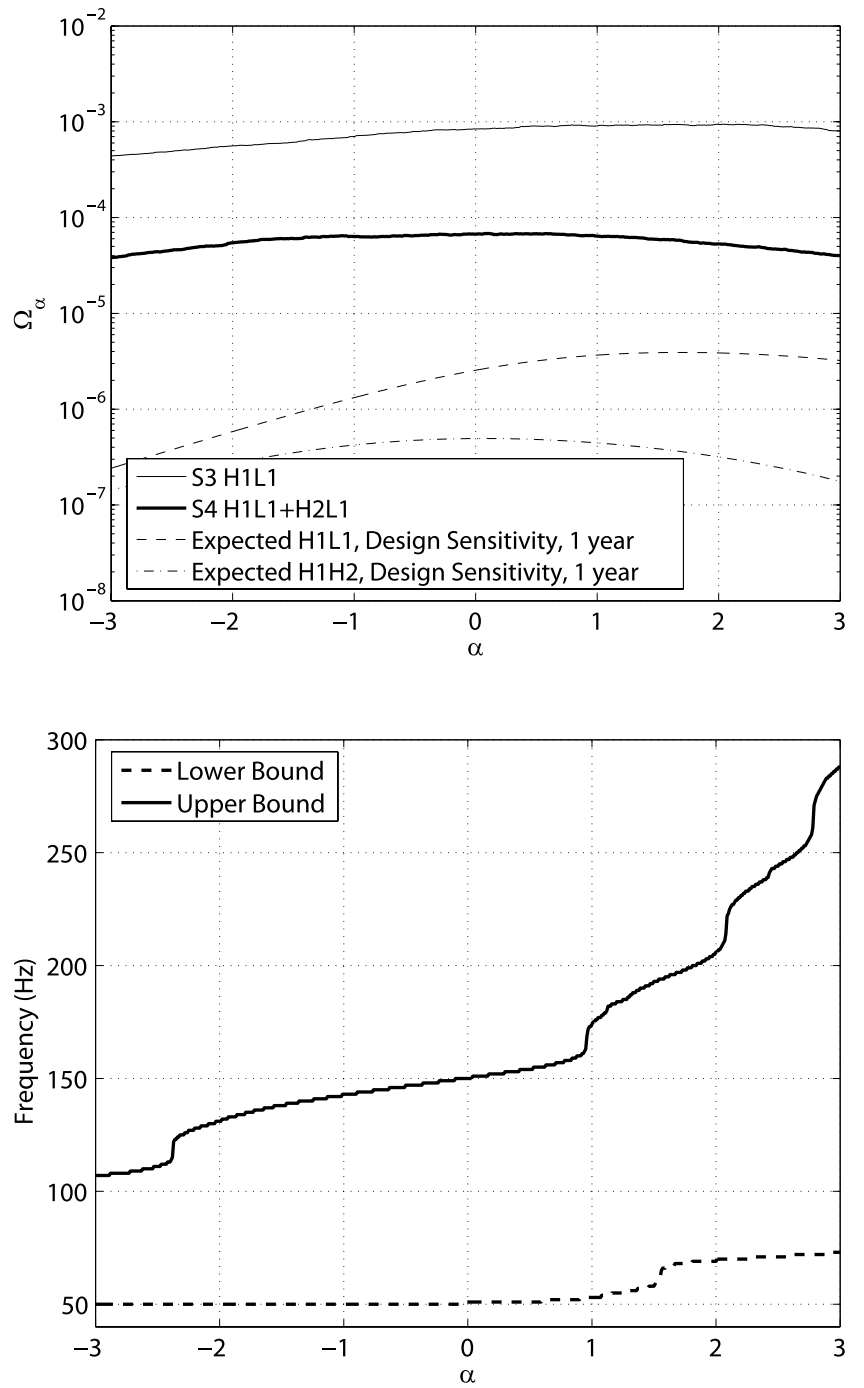

FIG. 11.-Top: $90 \%$ UL on $\Omega_{\alpha}$ as a function of $\alpha$ for S3 H1-L1 and S4 H1$\mathrm{L} 1+\mathrm{H} 2-\mathrm{L} 1$ combined and expected final sensitivities of LIGO H1-L1 and H1$\mathrm{H} 2$ pairs, assuming LIGO design sensitivity and $1 \mathrm{yr}$ of exposure. Bottom: Frequency band containing $99 \%$ of the full sensitivity (as determined by the inverse variance) is plotted as a function of $\alpha$ for the S4 result.

for injection 3. It also shows the inverse Fourier transform of the spectrum, which is equivalent to the estimate of $\Omega_{0}$ for different values of time lag between two interferometers (for short time lags).

We performed a sequence of software injections, where the injected signal is simply added to the interferometer data in the analysis. We performed 10 trials for 4 injection amplitudes using
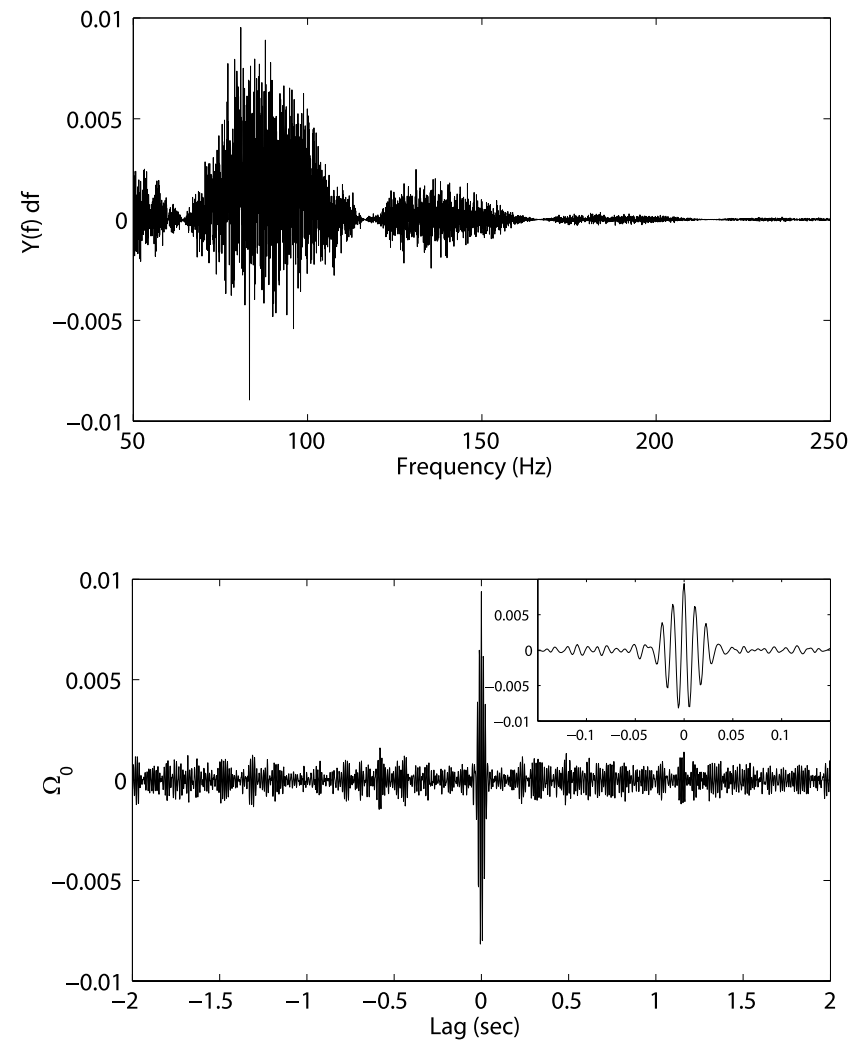

FIG. 12.-H1-L1 injection 3 (with intended injection amplitude $\Omega_{0}=1.1 \times$ $10^{-2}$ ). Top: Cross-correlation spectrum $Y(f)$. Bottom: Inverse Fourier transform of the cross-correlation spectrum indicates the clear signal at zero lag. The inset plot is a zoom-in around zero lag.

about $1 / 3$ of the $\mathrm{S} 4 \mathrm{H} 1-\mathrm{L} 1$ data. Figure 13 shows that the signal is successfully recovered down to $1 \times 10^{-4}$ in $\Omega_{0}$. Moreover, the theoretical error bars agree well with the standard deviation over the 10 trials. All injections were performed assuming a frequencyindependent $\mathrm{GW}$ spectrum $(\alpha=0)$.

\section{IMPLICATIONS}

In this section, we investigate the implications of the new upper limit for some of the models of the stochastic GW background. We also discuss the complementarity of our result with various other experimental constraints on the stochastic GW background.

\subsection{Complementarity with Other Measurements and Observations}

Figure 14 compares different experiments and some of the theoretical models. We note again our assumption regarding

TABLE 3

Summary of the Hardware Injection Amplitudes During S4

\begin{tabular}{|c|c|c|c|c|}
\hline $\begin{array}{l}\text { Injection } \\
\text { (1) }\end{array}$ & $\begin{array}{l}\text { H1-L1 Expected } \\
\left(\times 10^{-2}\right) \\
(2)\end{array}$ & $\begin{array}{l}\text { H1-L1 Recovered } \\
\qquad\left(\times 10^{-2}\right) \\
(3)\end{array}$ & $\begin{array}{l}\text { H2-L1 Expected } \\
\left(\times 10^{-2}\right) \\
(4)\end{array}$ & $\begin{array}{l}\text { H2-L1 Recovered } \\
\left(\times 10^{-2}\right) \\
(5)\end{array}$ \\
\hline 1 & 9.1 & $7.9 \pm 0.2 \pm 0.8$ & 7.9 & $6.9 \pm 0.4 \pm 0.6$ \\
\hline 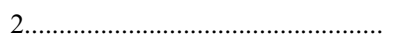 & 2.5 & $2.5 \pm 0.4 \pm 0.2$ & 2.3 & $1.5 \pm 0.6 \pm 0.2$ \\
\hline З & 1.1 & $0.95 \pm 0.04 \pm 0.10$ & $\ldots$ & $\ldots$ \\
\hline
\end{tabular}

Notes.-Cols. (2) and (4) indicate the expected injection amplitudes for H1-L1 and H2-L1, respectively, based on the signal injected into the differential-arm servo. Cols. (3) and (5) list the recovered values using the H1-L1 and H2-L1 pairs respectively. The recovered values are listed with statistical errors (as defined in eq. [4]) and with systematic errors (estimated using $5 \%$ calibration uncertainty in $\mathrm{L} 1$ and $8 \%$ calibration uncertainty in $\mathrm{H} 1$ and $\mathrm{H} 2$, added in quadrature). For injection 3, the data of $\mathrm{H} 2$ interferometer were compromised due to a failure of the interferometer's laser. 


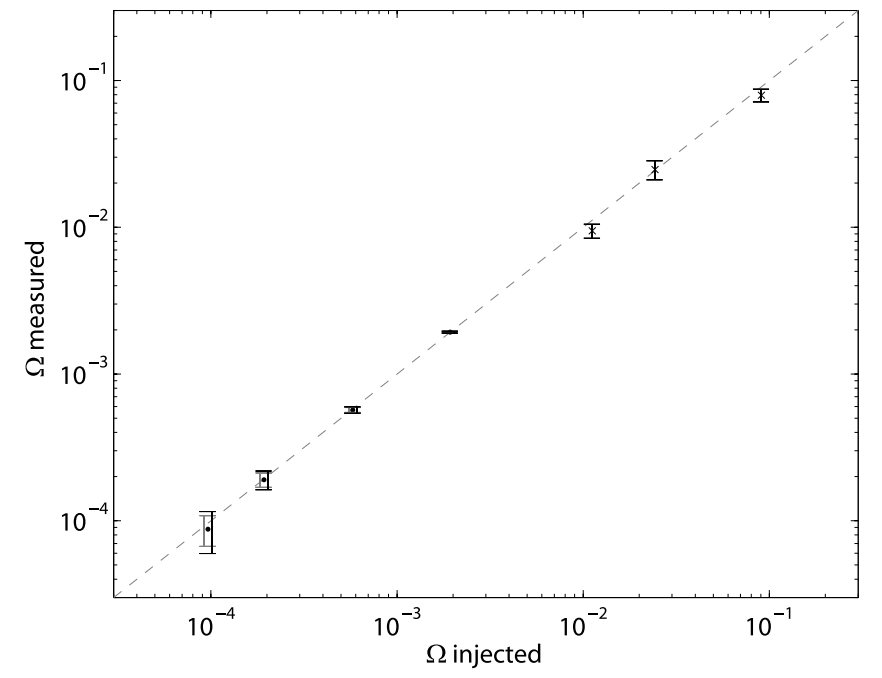

FIG. 13.- Injections using H1-L1 data; 10 trials were performed for software injections (denoted by dots) with amplitudes $\Omega_{0}=1 \times 10^{-4}, 2 \times 10^{-4}, 6 \times 10^{-4}$, and $2 \times 10^{-3}$. The left gray error bars denote the theoretical errors, while the right black error bars denote the standard errors over the 10 trials. The remaining points (denoted by crosses) correspond to the three hardware injections listed in Table 3; their error bars correspond to statistical and systematic errors added in quadrature, as shown in Table 3.

the Hubble parameter; when writing $\Omega_{\mathrm{GW}}$ we implicitly mean $\Omega_{\mathrm{GW}} \times\left[H_{0} /\left(72 \mathrm{~km} \mathrm{~s}^{-1} \mathrm{Mpc}^{-1}\right)\right]^{2}$.

For wavelengths larger than the horizon size at the surface of last scattering (redshifted to today, this corresponds to frequencies below $\sim 10^{-16} \mathrm{~Hz}$ ), the COBE observations of the CMB constrain the stochastic gravitational wave background to $\Omega_{\mathrm{GW}}(f)<7 \times 10^{-11}\left(3 \times 10^{-18} \mathrm{~Hz} / f\right)^{2}$ (see Allen \& Koranda 1994; Allen 1997). In the framework of standard inflationary models (Turner 1997), this constraint can be extrapolated to frequencies above $\sim 10^{-16} \mathrm{~Hz}: \Omega_{\mathrm{GW}}(f) \lesssim 10^{-14}$, with some dependence on the inflationary model parameters.

The fluctuations in the arrival times of millisecond pulsar signals can be used to place a $95 \%$ detection rate upper bound at $\sim 10^{-8} \mathrm{~Hz}$ (Jenet et al. 2006): $\Omega_{\mathrm{GW}}(f)<3.9 \times 10^{-8}$ (assuming frequency-independent GW spectrum). Similarly, Doppler tracking of the Cassini spacecraft can be used to arrive at yet another bound (Armstrong et al. 2003): $\Omega_{\mathrm{GW}}(f)<0.027$ at $1.2 \times 10^{-6} \mathrm{~Hz}$.

If the energy density carried by the gravitational waves at the time of big bang nucleosynthesis (BBN) were large, the amounts of the light nuclei produced in the process could be altered. Hence, the BBN model and observations can be used to constrain the total energy carried by gravitational waves at the time of nucleosynthesis (Kolb \& Turner 1990; Maggiore 2000; Allen 1997):

$$
\int \Omega_{\mathrm{GW}}(f) d(\ln f)<1.1 \times 10^{-5}\left(N_{\nu}-3\right),
$$

where $N_{\nu}$ (the effective number of neutrino species at the time of $\mathrm{BBN}$ ) captures the uncertainty in the radiation content during BBN. Measurements of the light-element abundances, combined with the Wilkinson Microwave Anistropy Probe (WMAP) data, give the following $95 \%$ upper bound: $N_{\nu}-3<1.4$ (Cyburt et al. 2005). This limit translates into $\int \Omega_{\mathrm{GW}}(f) d(\ln f)<1.5 \times 10^{-5}$. This bound applies down to $\sim 10^{-10} \mathrm{~Hz}$, corresponding to the horizon size at the time of BBN.

Gravitational waves are also expected to leave a possible imprint on the CMB and matter spectra, similar to that of massless

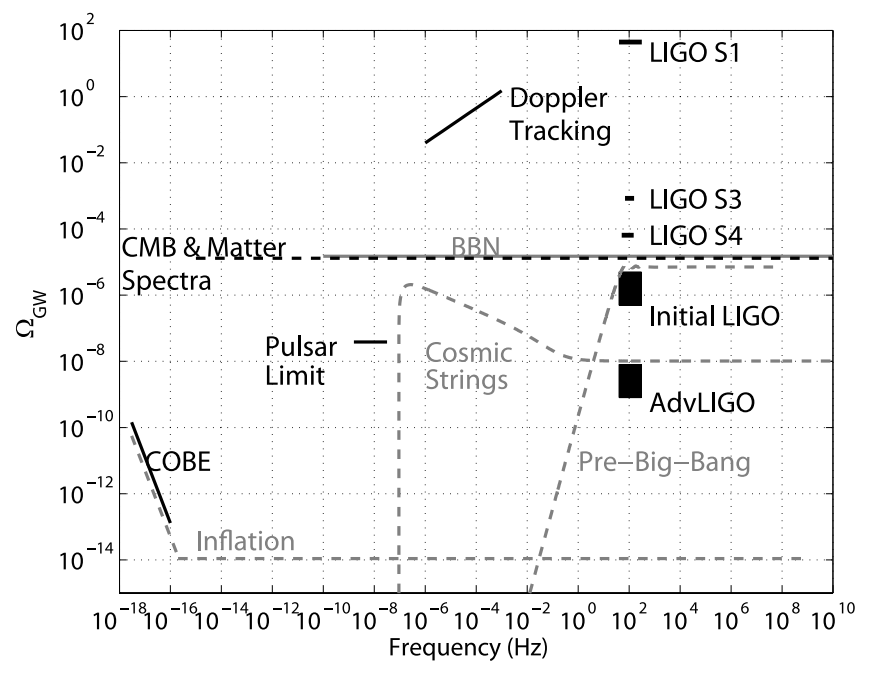

FIG. 14.-Landscape plot (see text for details). The curves corresponding to inflationary, cosmic-string, and pre-big bang models are examples; significant variations of the predicted spectra are possible as the model parameters are varied. The bounds labeled "BBN" and "CMB \& Matter Spectra" do not apply to $\Omega_{\mathrm{GW}}(f)$, but rather to the integral $\int \Omega_{\mathrm{GW}}(f) d(\ln f)$ over the frequency range spanned by the corresponding lines.

neutrinos. Smith et al. (2006b) used recent measurements of the CMB anisotropy spectrum, galaxy power spectrum, and Ly $\alpha$ forest to constrain the energy density carried by gravitational waves to $\int \Omega_{\mathrm{GW}}(f) d(\ln f)<1.3 \times 10^{-5}$ for homogeneous initial conditions at $95 \%$ confidence level. This bound is competitive with the BBN bound and it extends down to $\sim 10^{-15} \mathrm{~Hz}$, corresponding to the horizon size at the time of CMB decoupling. It is also expected to improve as new experiments come online (such as Planck or $C M B P o l$ ).

The LIGO results apply to the frequency region around $100 \mathrm{~Hz}$. The result discussed in this paper is an improvement by a factor of 13 over the previous LIGO result in the $100 \mathrm{~Hz}$ region, for a frequency-independent spectrum of GW background. A $1 \mathrm{yr}$ run at design sensitivity of LIGO (the S5 run, which began in November of 2005) is expected to improve the sensitivity by another factor of 10-100, while Advanced LIGO is expected to achieve sensitivities better by yet another factor of $100-1000$, eventually reaching $10^{-9}-10^{-8}$ for $\Omega_{0}$. The uncertainty in the final reach of LIGO and Advanced LIGO comes from the potential instrumental correlations that could be present between the collocated Hanford interferometers and from the uncertainty in the final optical configurations of interferometers in Advanced LIGO. The result discussed in this paper is still weaker than the indirect BBN bound (if one assumes that the gravitational-wave spectrum is limited to the LIGO frequency band), but future runs by LIGO and Advanced LIGO are expected to surpass this bound.

The standard inflationary models are most stringently constrained by the CMB bound at lowest frequencies. Although they are most likely out of range of LIGO and Advanced LIGO, they may be accessible to future GW interferometers (Smith et al. 2006a). However, there are models of stochastic GW background that LIGO is beginning to explore. We illustrate this with examples of cosmic string and pre-big bang models.

\subsection{Implications for Cosmic String Models}

Cosmic strings can be formed as linear defects during symmetrybreaking phase transitions in the early universe, as well as in string theory inspired inflation scenarios. In the latter case they have been dubbed cosmic superstrings. CMB data is not consistent 
with cosmic strings as the predominant source of density fluctuations in the universe. Their existence, however, is not ruled out below the grand unified theory (GUT) scale, and cosmic strings may still lead to a myriad of detectable astrophysical signatures, such as gravitational radiation, gamma-ray bursts, and ultrahigh energy cosmic rays. For a review, see Vilenkin \& Shellard (2000).

Damour \& Vilenkin (2005) investigated the stochastic background of gravitational waves produced by cusps on cosmic strings, integrated over all redshifts and all directions on the sky. They find that three parameters define the gravitational-wave spectrum due to cosmic strings:

1. String tension $\mu$.- This parameter is usually expressed as a dimensionless quantity $G \mu$ (assuming speed of light $c=1$ ), where $G$ is Newton's constant. String-theory-inspired inflation scenarios prefer the range $10^{-11} \leq G \mu \leq 10^{-6}$.

2. Reconnection probability p.-While ordinary, field-theoretic strings reconnect whenever they intersect $(p=1)$, the reconnection probability for superstrings is typically smaller than 1 . In particular, the theoretically favored range is $10^{-3} \leq p \leq 1$ (Jackson et al. 2005).

3. $\epsilon$.-This parameter describes the typical size of the closed loops produced in the string network. The value of this parameter is uncertain, and it can span several orders of magnitude. We consider the range $10^{-13}<\epsilon<1$, which is both theoretically viable and most interesting from the point of view of LIGO.

These parameters determine both the amplitude and the shape of the gravitational-wave spectrum. In particular, parameters $\epsilon$ and $G \mu$ determine the lowest frequency (at a given redshift) at which a string loop could emit gravitational radiation. Since there is a low-frequency cutoff to the predicted gravitational-wave spectrum, it is possible to have a cosmic string model that would avoid the low-frequency bounds due to CMB or pulsar timing measurements, but still be within reach of LIGO (cf. Fig. 14).

Figure 15 shows the region of the parameter space (for $p=$ $10^{-3}$ ) excluded by the result discussed in this paper and by the $\mathrm{S} 3$ result (Abbott et al. 2005), as well as the expected reach of LIGO and Advanced LIGO in future runs. As shown in this figure, LIGO is most sensitive to the regions of large $G \mu$ and small $\epsilon$. Moreover, LIGO results are complementary to the pulsar timing limit, which is most sensitive to models with large $G \mu$ and large $\epsilon$, and to the indirect BBN limit. In particular, the population of models with $p=10^{-3}, \epsilon \lesssim 5 \times 10^{-11}$, and $5 \times 10^{-9} \lesssim G \mu \lesssim 7 \times$ $10^{-8}$ are excluded by the result discussed in this paper, but are not accessible to other current experimental bounds. To produce this figure, we used equations (4.1)-(4.7) from Damour \& Vilenkin (2005).

We note that as $p$ increases toward 1 , the bounds/reach of all experiments in the $G \mu-\epsilon$ plane weaken because the amplitude of the gravitational-wave spectrum scales with $1 / p$. We also note that there are significant uncertainties in the calculation discussed by Damour \& Vilenkin (2005), mostly due to incomplete understanding of the string network behavior. Recent numerical simulations of cosmic string networks (Martins \& Shellard 2006; Vanchurin et al. 2006; Ringeval et al. 2005) suggest that loop sizes are related to the large-scale dynamics of the network. In this case, the expected stochastic background at the frequencies of pulsar timing experiments could be substantially larger (Hogan 2006). In addition, the treatment of Damour \& Vilenkin (2005) did not account for the effects of late-time acceleration. Thus, a more detailed analysis of the stochastic background produced by cosmic strings is necessary.

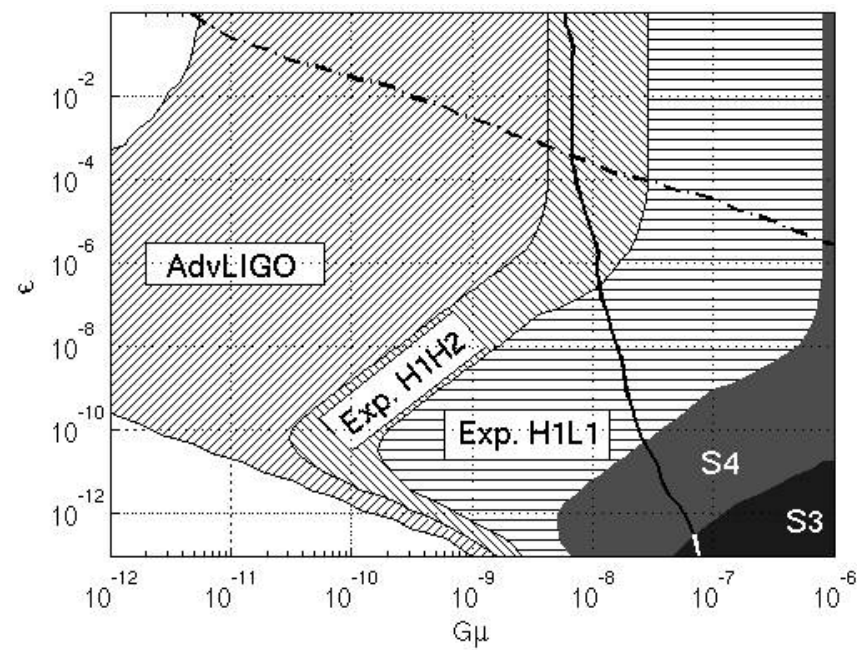

FIG. 15.-The $\epsilon-G \mu$ plane for the cosmic string models with $p=10^{-3}$. The shaded regions are excluded by the LIGO S3 limit (dark gray) and by the LIGO $\mathrm{S} 4$ limit presented here (light gray). The hatched regions are accessible to future LIGO runs: expected LIGO sensitivity for the H1-L1 pair, assuming design interferometer strain sensitivity and 1 yr of exposure (horizontal hatching); expected LIGO sensitivity for the $\mathrm{H} 1-\mathrm{H} 2$ pair, assuming design interferometer strain sensitivity and 1 yr of exposure ( || hatching); expected Advanced LIGO sensitivity for the $\mathrm{H} 1-\mathrm{H} 2$ pair, assuming interferometer strain sensitivity tuned for the binary neutron star inspiral search and $1 \mathrm{yr}$ of exposure (/// hatching). The dash-dotted black curve is the exclusion curve based on the pulsar limit (Jenet et al. 2006; the excluded region is above the curve). The solid black curve is the exclusion curve based on the indirect big bang-nucleosynthesis bound (the excluded region is to the right of the curve).

\subsection{Implications for Pre-Big Bang Models}

Pre-big bang models are cosmology models motivated by string theory (Gasperini \& Veneziano 1993, 2003). In these models, the universe evolves through several phases: "dilation" phase in which the universe is large and shrinking; "stringy" phase in which the curvature of the universe is high; and standard radiation- and matter-dominated phases. The GW spectrum is generated by amplification of vacuum fluctuations as the universe transitions from one phase to another. The shape and amplitude of the spectrum are determined by the states of the universe in the different phases. Although the "stringy phase" of the model and the transition to the radiation phase are not well understood, some models have been proposed in the literature that may partially describe it. In the formalism developed by Buonanno et al. (1997), the GW spectrum produced by the model can be described as

$$
\text { 1. } \Omega_{\mathrm{GW}}(f) \sim f^{3} \text { for } f<f_{s} \text {, where } f_{s} \text { is essentially }
$$
unconstrained.

2. $\Omega_{\mathrm{GW}}(f) \sim f^{3-2 \mu}$ for $f_{s}<f<f_{1}$, where $\mu<1.5$ defines the evolution of the universe in the "stringy" phase. The cutoff frequency $f_{1}$ is determined by string-related parameters and its most natural value is expected to be $4.3 \times 10^{10} \mathrm{~Hz}$.

Following the analysis in Mandic \& Buonanno (2006), we scan the parameter space $\left(f_{s}, \mu, f_{1}\right)$. For each set of values of these parameters, we calculate the GW spectrum following Buonanno et al. (1997) and check whether the model is accessible to the current (and past) LIGO results. We also project the sensitivity of initial and Advanced LIGO to these models. Figure 16 shows the $f_{1}-\mu$ plane for $f_{s}=30 \mathrm{~Hz}$. Note that the LIGO S3 and S4 results are beginning to explore the parameter space of these models, although the indirect BBN bound is still a stronger constraint. Future runs of LIGO and of Advanced 


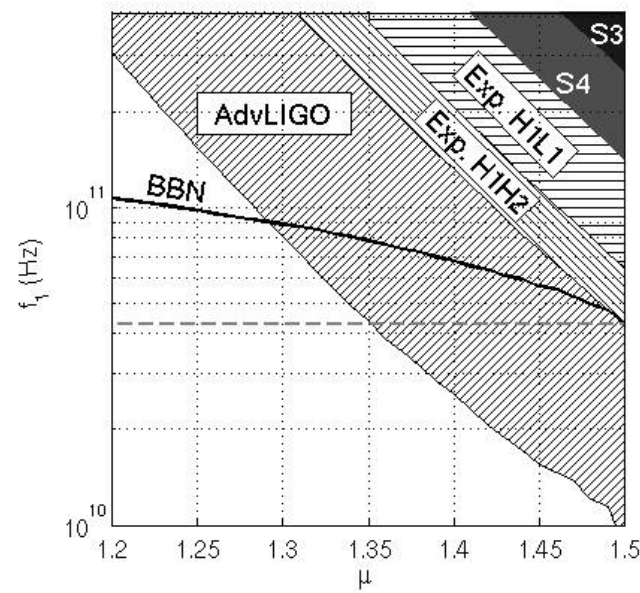

FIG. 16.-The $f_{1}-\mu$ plane for the pre-big bang models with $f_{s}=30 \mathrm{~Hz}$. The shaded regions are excluded by the LIGO S3 limit (dark gray) and by the LIGO S4 limit presented here (light gray). The hatched regions will be accessible to future LIGO runs: expected LIGO sensitivity for the H1-L1 pair, assuming design interferometer strain sensitivity and 1 yr of exposure (horizontal hatching); expected LIGO sensitivity for the $\mathrm{H} 1-\mathrm{H} 2$ pair, assuming design interferometer strain sensitivity and 1 year of exposure (III hatching); expected Advanced LIGO sensitivity for the $\mathrm{H} 1-\mathrm{H} 2$ pair, assuming interferometer strain sensitivity tuned for the binary neutron star inspiral search and $1 \mathrm{yr}$ of exposure (/// hatching). The solid black curve is the exclusion curve based on the BBN limit (the excluded region is above the curve). The horizontal dashed line denotes the most natural value of $f_{1}=4.3 \times 10^{10} \mathrm{~Hz}$.

LIGO are expected to explore significantly larger parts of the parameter space, eventually surpassing the BBN bound (in some parts of the parameter space) and even reaching the most natural value of $f_{1}=4.3 \times 10^{10} \mathrm{~Hz}$.

\section{CONCLUSIONS}

LIGO data acquired during the science run S4 yield a new Bayesian $90 \%$ upper limit on the amplitude of the stochastic GW background: $\Omega_{0}<6.5 \times 10^{-5}$ for the frequency-independent GW spectrum $(\alpha=0)$ in the frequency band $51-150 \mathrm{~Hz}$. Similar limits are obtained for other values of $\alpha$, as shown in Figure 11. This result is an improvement by a factor of 13 over the previous upper limit in the same frequency range, obtained by LIGO in the science run $\mathrm{S} 3$.
This result is obtained using $192 \mathrm{~s}$ long intervals of data with $1 / 32 \mathrm{~Hz}$ frequency resolution of the spectra, and it properly excludes the known instrumental correlations at $1 \mathrm{~Hz}$ harmonics. It is fully consistent with the blind result that uses $60 \mathrm{~s}$ long intervals of data with $1 / 4 \mathrm{~Hz}$ frequency resolution, which is slightly contaminated by the instrumental $1 \mathrm{~Hz}$ harmonics. It is also more conservative than the blind result, as the theoretical error is larger due to the smaller amount of data available in the form of acceptable $192 \mathrm{~s}$ intervals (as compared to the $60 \mathrm{~s}$ intervals).

This result is complementary to the constraints on the gravitational-wave spectrum, based on the measurements of the $\mathrm{CMB}$ spectrum and pulsar timing. It is still weaker than the indirect BBN bound for models where the stochastic gravitationalwave background is limited to the LIGO frequency band. The ongoing $1 \mathrm{yr}$ long run of LIGO at the design sensitivity, and the future runs of Advanced LIGO, are expected to surpass the BBN bound for such models. Furthermore, this result is already exploring the parameter space of some models of the stochastic GW background, such as cosmic strings models and pre-big bang models.

The authors gratefully acknowledge the support of the United States National Science Foundation for the construction and operation of the LIGO Laboratory and the Particle Physics and Astronomy Research Council of the United Kingdom, the MaxPlanck-Society, and the State of Niedersachsen/Germany for support of the construction and operation of the GEO600 detector. The authors also gratefully acknowledge the support of the research by these agencies and by the Australian Research Council, the Natural Sciences and Engineering Research Council of Canada, the Council of Scientific and Industrial Research of India, the Department of Science and Technology of India, the Spanish Ministerio de Educacion y Ciencia, The National Aeronautics and Space Administration, the John Simon Guggenheim Foundation, the Alexander von Humboldt Foundation, the Leverhulme Trust, the David and Lucile Packard Foundation, the Research Corporation, and the Alfred P. Sloan Foundation. LIGO DCC number: P060012-07-D.

\section{REFERENCES}

Abbott, B., et al. 2004a, Nucl. Instrum. Methods Phys. Res. A, 517, 154 2004b, Phys. Rev. D, 69, 122004 2005, Phys. Rev. Lett., 95, 221101

Allen, B. 1997, in Relativistic Gravitation and Gravitational Radiation, ed. J.-A. Marck \& J.-P. Lasota (Cambridge: Cambridge Univ. Press), 373

Allen, B., \& Koranda, S. 1994, Phys. Rev. D, 50, 3713

Allen, B., \& Romano, J. 1999, Phys. Rev. D, 59, 102001

Apreda, R., Maggiore, M., Nicolis, A., \& Riotto, A. 2002, Nucl. Phys. B, 631, 342

Armstrong, J. W., et al. 2003, ApJ, 599, 806

Astone, P., et al. 1999, A\&A, 351, 811

Baskaran, D., Grishchuk, L. P., \& Polnarev, A. G. 2006, Phys. Rev. D, 74, 083008

Bendat, J. S., \& Piersol, A. G. 2000, Random Data Analysis and Measurement Procedures (3rd ed.; New York: Wiley)

Bennet, C. L., et al. 2003, ApJS, 148, 1

Bose, S., et al. 2003, Classical Quantum Gravity, 20, S677

Buonanno, A., Maggiore, M., \& Ungarelli, C. 1997, Phys. Rev. D, 55, 3330

Caldwell, R. R., \& Allen, B. 1992, Phys. Rev. D, 45, 3447

Cooray, A. 2004, MNRAS, 354, 25

Coward, D. M., Burman, R. R., \& Blair, D. G. 2002, MNRAS, 329, 411

Cyburt, R. H., Fields, B.D, Olive, K. A., \& Skillman, E. 2005, Astropart. Phys., 23,313
Damour, T., \& Vilenkin, A. 2000, Phys. Rev. Lett., 85, 3761 2005, Phys. Rev. D, 71, 063510

Fotopoulos, N. 2006, Classical Quantum Gravity, 23, S693

Gasperini, M., \& Veneziano, G. 1993, Astropart. Phys., 1, 317 2003, Phys. Rep., 373, 1

Grishchuk, L. P. 1975, Soviet Phys.-JETP, 40, 409 1997, Classical Quantum Gravity, 14, 1445

Grishchuk, L. P., et al. 2006, LIGO Technical Note T060270-00-Z (Pasadena:

California Inst. Tech.), http://www.ligo.caltech.edu/docs/T/T060270-00.pdf Hogan, C. J. 2006, Phys. Rev. D, 74, 043526

Jackson, M. G., Jones, N. T., \& Polchinski, J. 2005, J. High Energy Phys., 10, 013

Jenet, F. A., et al. 2006, ApJ, 653, 1571

Kolb, E., \& Turner, M. S. 1990, The Early Universe (Reading: Addison-Wesley) Kosowsky, A., Turner, M. S., \& Watkins, R. 1992, Phys. Rev. Lett., 69, 2026 Lazzarini, A., \& Romano, J. 2004, LIGO Technical Note T040089-00-Z (Pasadena: California Inst. Tech.), http://www.ligo.caltech.edu/docs/ T/T040089-00.pdf

Loredo, T. J. 1990, in Maximum Entropy and Bayesian Methods, ed. P. F. Fougère (Dordrecht: Kluwer), 81

Maggiore, M. 2000, Phys. Rep., 331, 283

Mandic, V., \& Buonanno, A. 2006, Phys. Rev. D, 73, 063008

Martins, C. J. A. P., \& Shellard, E. P. S. 2006, Phys. Rev. D, 73, 043515

Regimbau, T., \& de Freitas Pacheco, J. A. 2001, A\&A, 376, 381 
Ringeval, C., Sakellariadou, M. \& Bouchet, F. 2005, preprint (astro-ph/0511646) Smith, T. L., Peiris, H. V., \& Cooray, A. 2006a, Phys. Rev. D, 73, 123503

Smith, T. L., Pierpaoli, E., \& Kamionkowski, M. 2006b, Phys. Rev. Lett., 97, 021301

Starobinsky, A. A. 1979, Pis'ma Zh. Eksp. Teor. Fiz., 30, 719
Turner, M. S. 1997, Phys. Rev. D, 55, 435

Vanchurin, V., Olum, K. D., \& Vilenkin, A. 2006, Phys. Rev. D, 74, 063527

Vilenkin, A., \& Shellard, E. P. S. 2000, Cosmic Strings and Other Topological Defects (Cambridge: Cambridge Univ. Press) 